
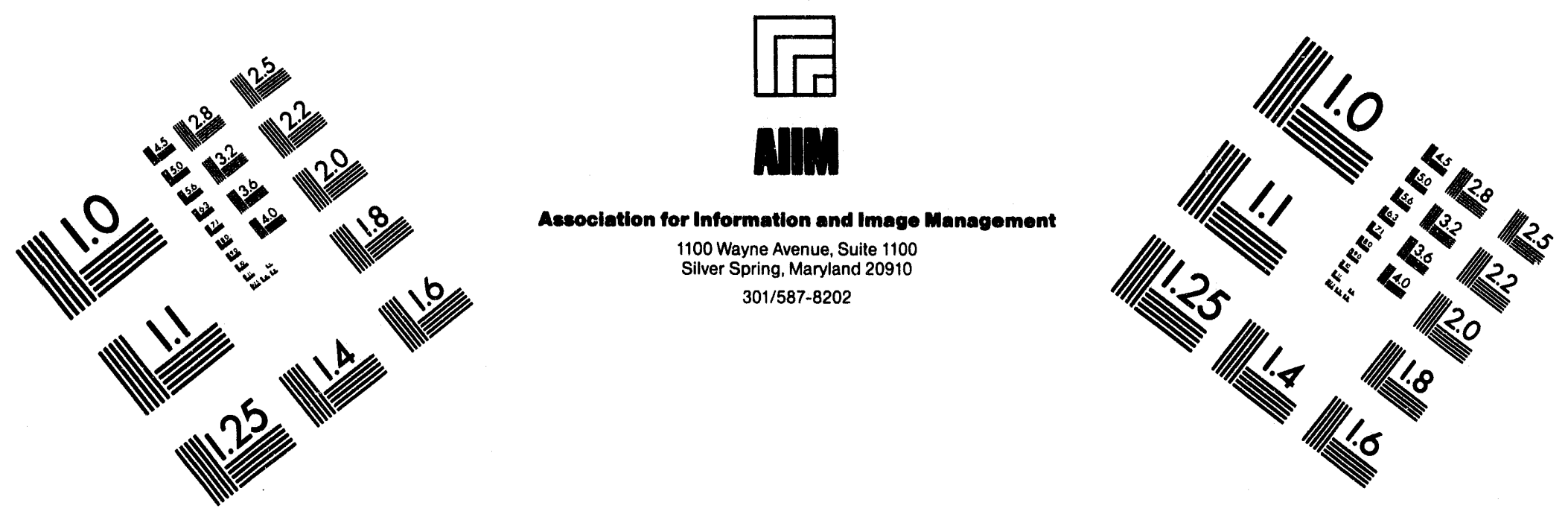

\title{
Centimeter
}

|m Inches
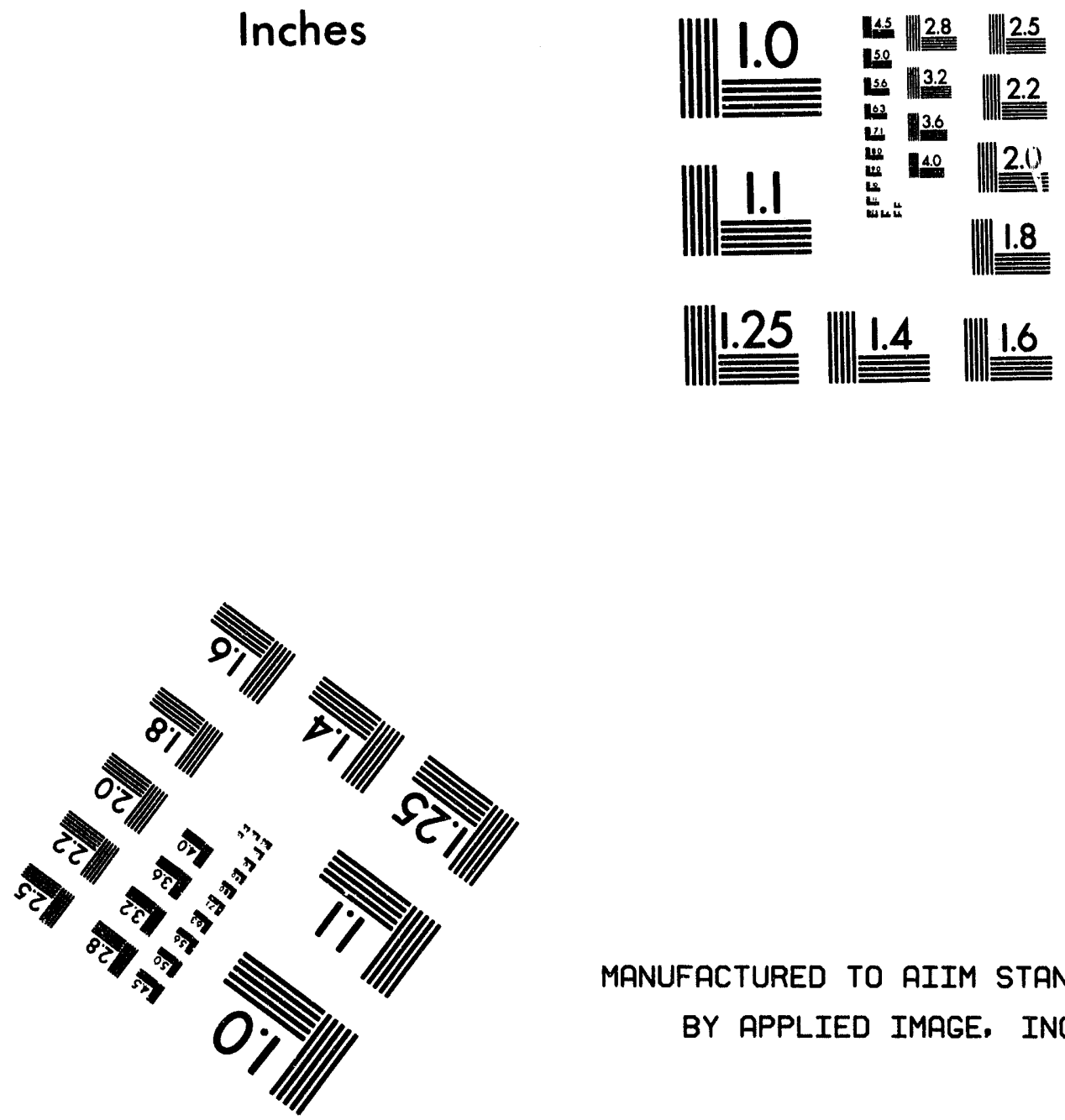

MANUFACTURED TO AIIM STANDARDS BY APPLIED IMAGE, INC.

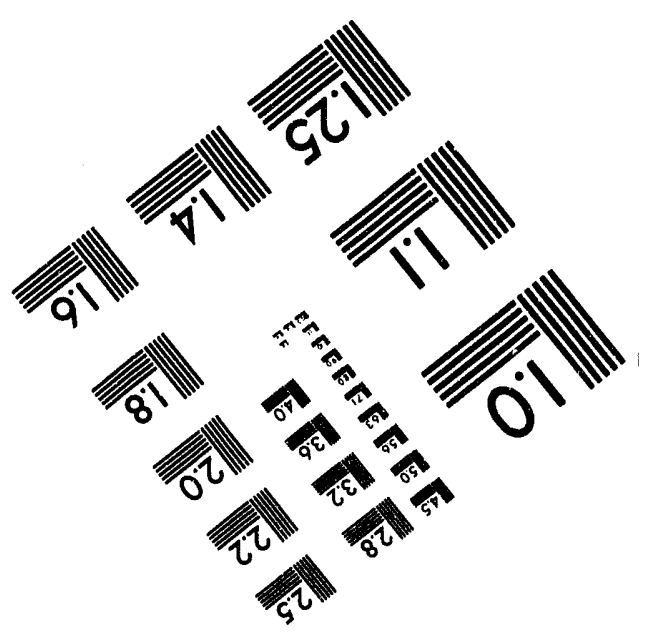



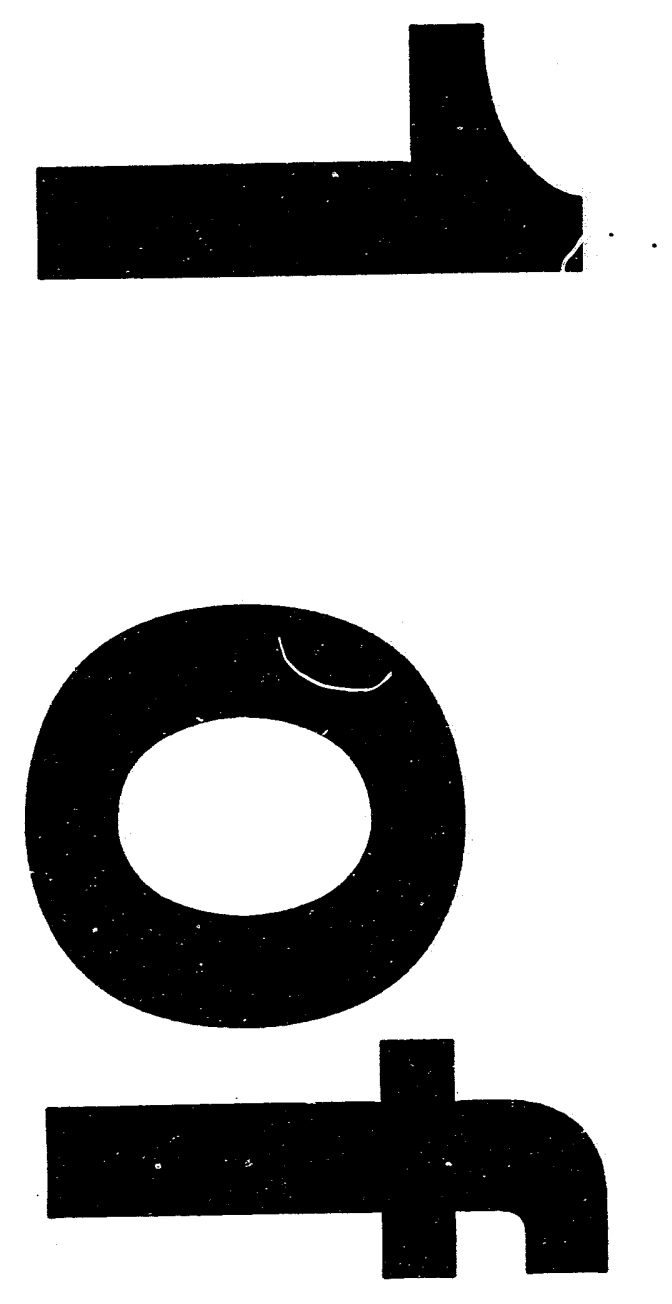

,'

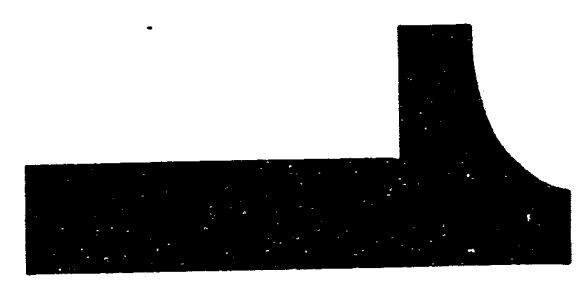


ANL/ESD/TM-65 $\sqrt{ }$

\section{Uptake of Explosives from Contaminated Soil by Existing Vegetation at the Joliet Army Ammunition Plant}

by J.F. Schneider, N.A. Tomczyk, S.D. Zellmer, and W.L. Banwart*

Reclamation Engineering and Geosciences Section, Energy Systems Division, Argonne National Laboratory, 9700 South Cass Avenue, Argonne, Illinois 60439

January 1994

Work sponsored by United States Department of Defense, U.S. Army Environmental Center

"Banwart is affiliated with the Agronomy Department, University of Illinois, Champaign-Urbana, Illinois.

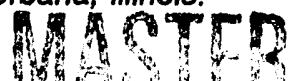

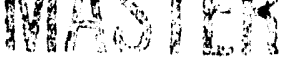

DISTRIEUTION OF THIS DOCUMENT IS UNLHMITED 


\section{Contents}

Acknowledgments............................................................... iv

Abstract....................................................................... 1

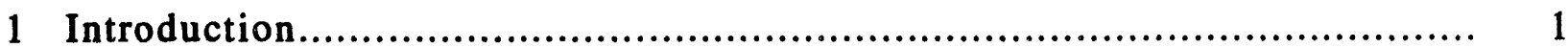

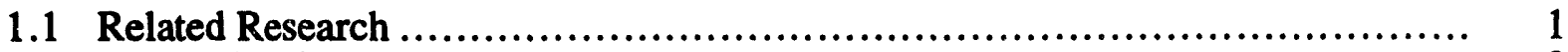

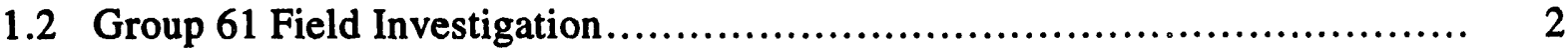

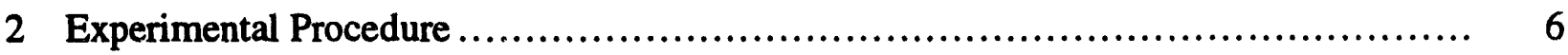

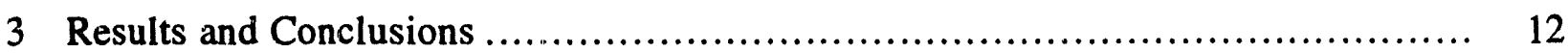

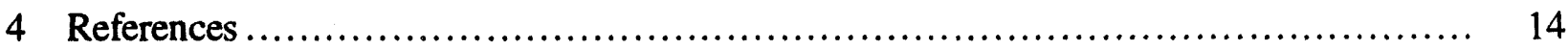

Appendix A: Analytical Chemistry Data from Previous Sampling Events at Group 61 ...................................................... 15

Appendix B: Summary of Analysis for Explosives from Previous

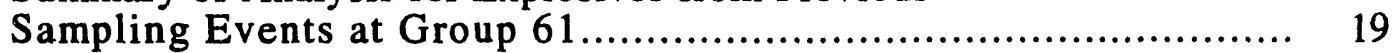

Appendix C: Documentation of Analytical Method for Explosives Analysis............ 23

\section{Figures}

1 Location of Group 61 at the Joliet Army Ammunition Plant.................... 3

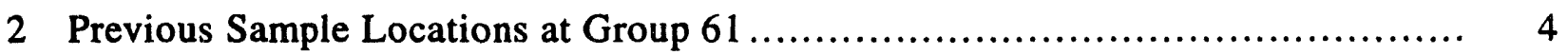

3 TNT Sample Locations at the Joliet Army Ammunition Plant, Group 61 - Ridge and Furrow Area.

4 TCLP Sample Locations at the Joliet Army Ammunition Plant, Group 61 - Ridge and Furrow Area........................................ 10

5 Chromatogram of Standard Explosives Mix..................................... 13

\section{Tables}

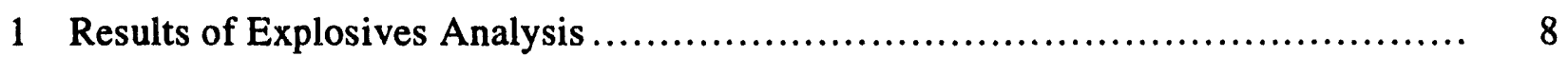

2 TCLP Results.............................................................. 11 


\section{Acknowledgments}

The authors would like to acknowiedge Diejun Chen for analyses of soil and plant samples, Ray Hinchman for expert plant identification, and the U.S. Army Environmental Center for funding this study. 


\title{
Uptake of Explosives from Contaminated Soil by Existing Vegetation at the Joliet Army Ammunition Plant
}

by

J.F. Schneider, N.A. Tomczyk, S.D. Zellmer, and W.L. Banwart

\begin{abstract}
This study examines the uptake of explosives by existing vegetation growing in TNT-contaminated soils on Group 61 at the Joliet Army Ammunition Plant (JAAP). The soils in this group were contaminated more than 40 years ago. In this study, existing plant materials and soil from the root zone were sampled from 15 locations and analyzed to determine TNT uptake by plants under natural field conditions. Plant materials were separated by species if more than one species was present at a sampling location. Standard methods were used to determine concentrations of explosives, their derivatives, and metabolites in the soil samples. Plant materials were also analyzed. No explosives were detected in the aboveground portion of any plant sample. However, the results indicate that TNT, 2-amino DNT, and/or 4-amino DNT were found in some root samples of false boneset (Kuhnia eupatorioides), teasel (Dipsacus sylvestris), and bromegrass (Bromus inermis). It is possible that slight soil contamination remained on the roots, especially in the case of the very fine roots for species like bromegrass, where washing was difficult. The presence of 2-amino DNT and 4-amino DNT, which could be plant metabolites of TNT, increases the likelihood that explosives were taken up by plant roots, as opposed to their presence resulting from external soil contamination.
\end{abstract}

\section{Introduction}

\subsection{Related Research}

Limited information has been published on the uptake of explosives from contaminated soils by plants. Palazzo and Leggett (1986) investigated the uptake of 2,4,6-trinitrotoluene (TNT) by yellow nutsedge grown in hydroponic solutions. They discovered that the condition of plants (weight, height, length) was affected by the presence of TNT in the solution, but when the concentration was increased from 5 to $20 \mathrm{mg} / \mathrm{L}$, they observed no significant difference in the effects. As the initial TNT concentration was increased, however, the metabolites 2-amino- and 4-aninodinitrotoluene (2-ADNT and 4-ADNT) also increased, with 4-ADNT produced more than three times as often as 2-ADNT in all parts of the plant except the leaves. Because no metabolites 
were detected in the solution, the authors believe that the plants were producing the metabolites. Folsom et al. (1988) and Pennington (1988) also studied TNT uptake by nutsedge from soils amended with TNT. Results of these studies show minimal uptake of TNT by the leafy portions of the plants.

Harvey et al. (1991) examined uptake of TNT by bush beans grown in hydroponic solutions containing $10 \mathrm{mg} / \mathrm{L}$ of TNT. Results of this study showed low concentrations of TNT $(0.2$ to $0.3 \mathrm{mg} / \mathrm{kg})$ in leaf material but much higher concentrations of TNT $(6.0$ to $7.0 \mathrm{mg} / \mathrm{kg})$ in root material. A study in hydroponics that used radio-labeled TNT (Harvey et al. 1990) showed uptake of TNT into the roots, with some radio-label deposition in stem and leaf tissue as well. Chromatographic results showed that aminodinitrotoluene isomers (2-ADNT and 4-ADNT) were found in root tissue, but investigators were unsure of their origin. Possible explanations for the isomers' presence were that the roots drew the isomers up from the solution or that the TNT was metabolized by either the root tissue or the microorganisms associated with the root tissue. Harvey et al. (1993) also showed uptake of tetryl by plants in a hydroponics study that used radio-labeled tetryl. The greater portion of tetryl was found in the root tissue, with less in the stems and still less in the leaves. The study also showed evidence of tetryl being metabolized to polar compounds.

Banwart et al. (1991) examined the uptake, in the greenhouse, of 1,3,5-trinitro-1,3,5triazine (RDX) by corn, soybeans, sorghum, and wheat, from soils spiked with four levels of RDX. Results of their investigation showed that concentrations of RDX in plant materials increased as RDX levels in the soil increased. The investigation determined the uptake of .explosives by plants grown in hydroponic solutions or grown in soils amended with explosives under greenhouse conditions. The potential for explosives entering the food chain through uptake by plants under field conditions has not been documented.

\subsection{Group 61 Field Investigation}

This study examines the uptake of explosives by existing vegetation growing in TNTcontaminated soils on Group 61 at the Joliet Army Ammunition Plant (JAAP). JAAP is a government-owned, contractor-operated U.S. Army industrial facility encompassing 23,544 acres of prime agricultural land in Will County, approximately $17 \mathrm{mi}$ south of Joliet, Illinois. Group 61 is located in the north-central portion of the Load-Assemble-Package (LAP) area of JAAP, covers approximately 80 acres, and was constructed in 1941 as part of the installation (Figure 1). Group 61 facilities were originally used for crystallizing ammonium nitrate, but they were extensively modified in 1945 to reclaim TNT from high-explosive shells. The reclamation operation involved the removal and recycling of explosives and a shell washout operation. Process water (pink water) from the washout operation was collected in a large sump. Overflow from the sump was disposed of by infiltration and evaporation in a 4-acre ridge-and-furrow area (Figure 2). 


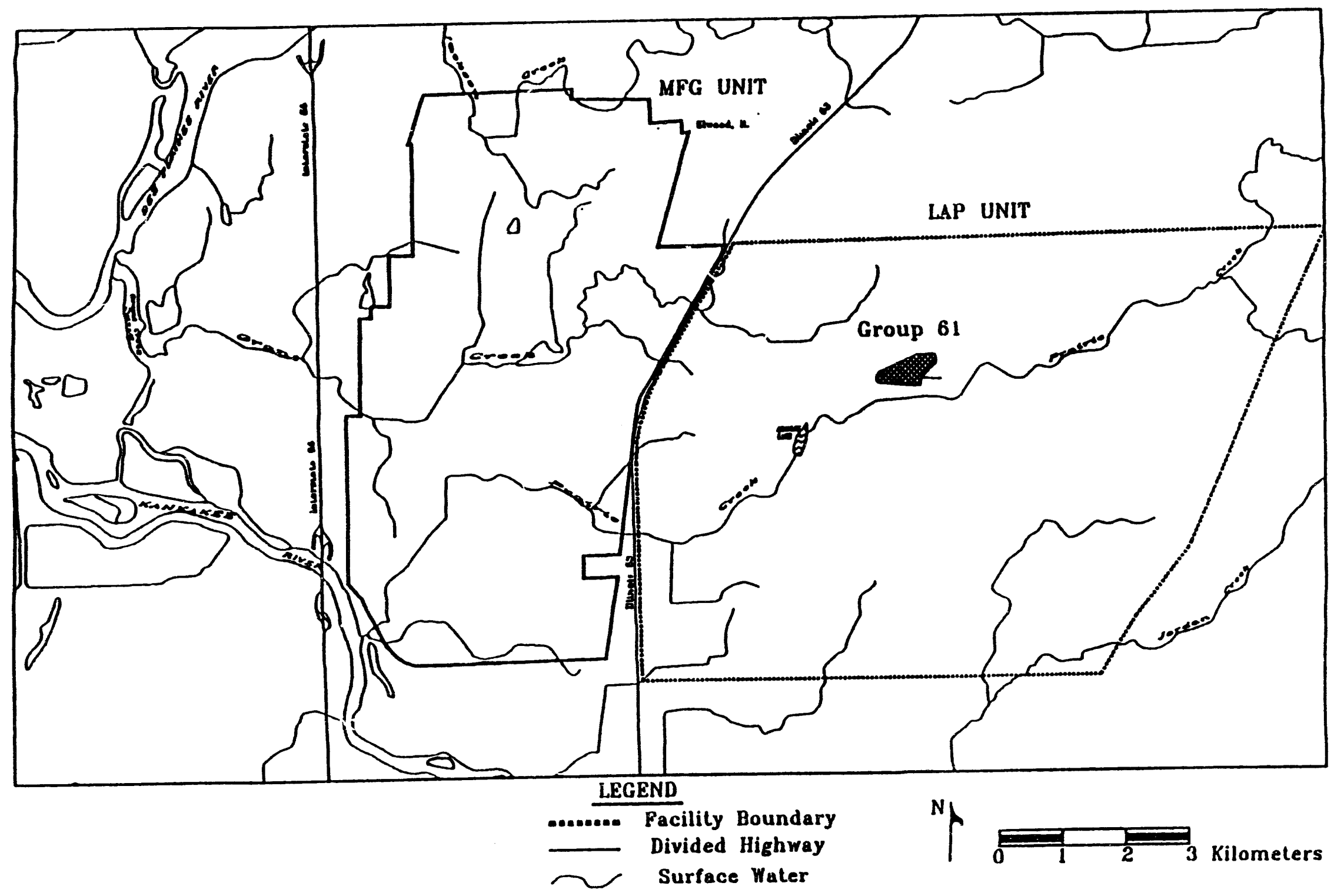

FIGURE 1 Location of Group 61 at the Joliet Army Ammunition Plant 


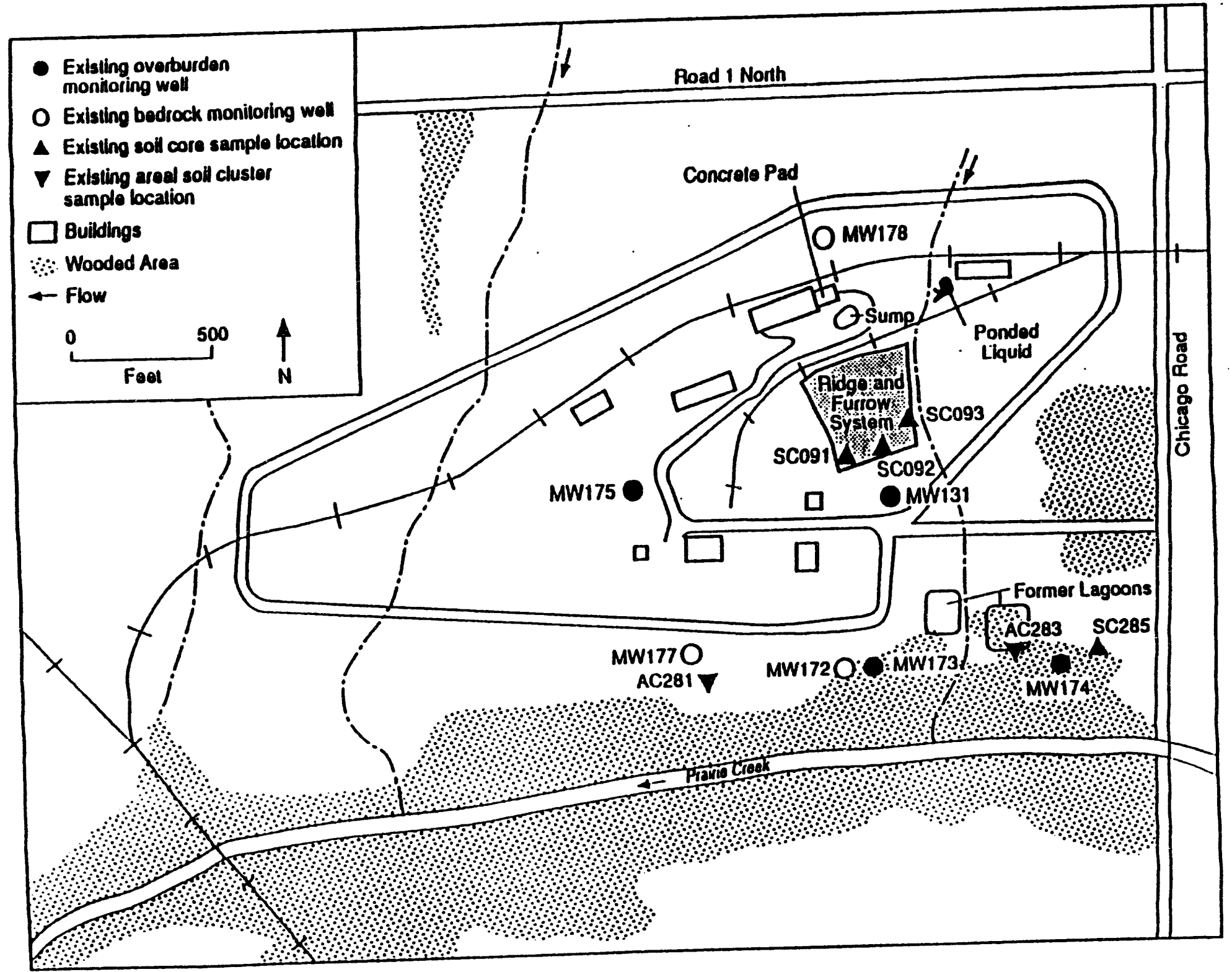

FIGURE 2 Previous Sample Locations at Group 61 (Source: Adapted from Ritchie et al. 1991) 
In the years following the closure of Group 61 facilities, erosion partially leveled the ridges and filled the furrows of the ridge-and-furrow area. Furrows on 7-ft centers are still evident, varying from 0 to $8 \mathrm{in}$. deep, depending on the degree of erosion. Plant cover, consisting of smooth bromegrass, other grasses, and forbs, has developed on the eastern portion of the site. Vegetative cover has developed on the ridges, but the presence of TNT in some areas of the furrow surface soil is evident by a reddish color and a lack of vegetation. The surface soil of the western portion of the site is rocky and has the appearance of subsoil or glacial till. Vegetative cover in this portion of the site is sparse and consists mainly of forbs. The lack of plant cover in this portion of the ridge-and-furrow area may result from high concentrations of TNT in the soil, low soil fertility, or poor physical condition of the soil. The soil in Group 61 was contaminated with TNT more than 40 years ago. In this study, existing plant materials and soil from the root zone were sampled and analyzed to determine TNT uptake by plants under natural field conditions. 


\section{Experimental Procedure}

Fiftren sampling locations were selected within the ridge-and-furrow area at Group 61 at JAAP (Figure 3). Sampling locations were divided into three groups, with five locations in each group, representing high, intermediate, and low levels of soil TNT contamination. (The level of contamination has been determined by previous studies; see Appendix A, adapted from Dames \& Moore 1991.) Soil samples were taken to confirm the level of contamination (Appendix B). Soil from the root zone and existing vegetative materials were collected from each location. Plant materials were separated by species if more than one species was present at a sampling location (see Table 1). Standard U.S. Army Toxic and Hazardous Materials Agency (USATHAMA) methods were used to determine concentrations of explosives, their derivatives, and metabolites in the soil samples (see Appendix C). All analyses were performed by Wayne Banwart (University of Illinois at Champaign-Urbana), who is USATHAMA-certified. ${ }^{1}$ Plant materials were analyzed to determine concentrations of explosives, their derivatives, and metabolites by using the methods described below. Additional soil samples were collected from random locations in the ridge-andfurrow area to characterize the toxicity of the soil by means of the U.S. Environmental Protection Agency (EPA) toxic characteristic leaching procedure (TCLP).

At each selected sampling location, vegetation was clipped to about 1 in. above the soil surface. Care was taken to prevent contact of the plant material with the soil and not to include plant materials that had been in direct contact with the soil in the plant samples. Plant materials were separated by species during collection. Roots were analyzed separately from the aboveground portions of the plants. Previous studies have shown that rinsing with distilled water removes surface TNT contamination from plant material. Root samples were washed with a mechanical elutriator in an attempt to remove any surface TNT contamination.

USATHAMA-approved high-pressure liquid chromatographic (HPLC) methods were employed to determine concentrations of explosives and their derivatives in soil at each sampling location. A method developed by Banwart and Hassett (1990), involving extraction, cleanup, and HPLC with ultraviolet detection, was used to analyze plant materials. The following procedure was used to determine presence of and concentrations of TNT in plant material:

1. Plant samples are dried at $26^{\circ} \mathrm{C}$. One-half gram of ground plant sample (60 mesh) is weighed into a $25-\mathrm{mL}$ Corvex tube equipped with a Teflon-lined screw cap.

2. Ten milliliters of dichloromethane is added to each tube. The tubes are placed in a water bath with cooling water and sonicated for 12 to $15 \mathrm{~h}$.

1 Wynne, D.J., Technical Support Division, U.S. Army Environmental Center, Aberdeen, Md., personal communication to W.L. Banwart, March 5, 1993. 


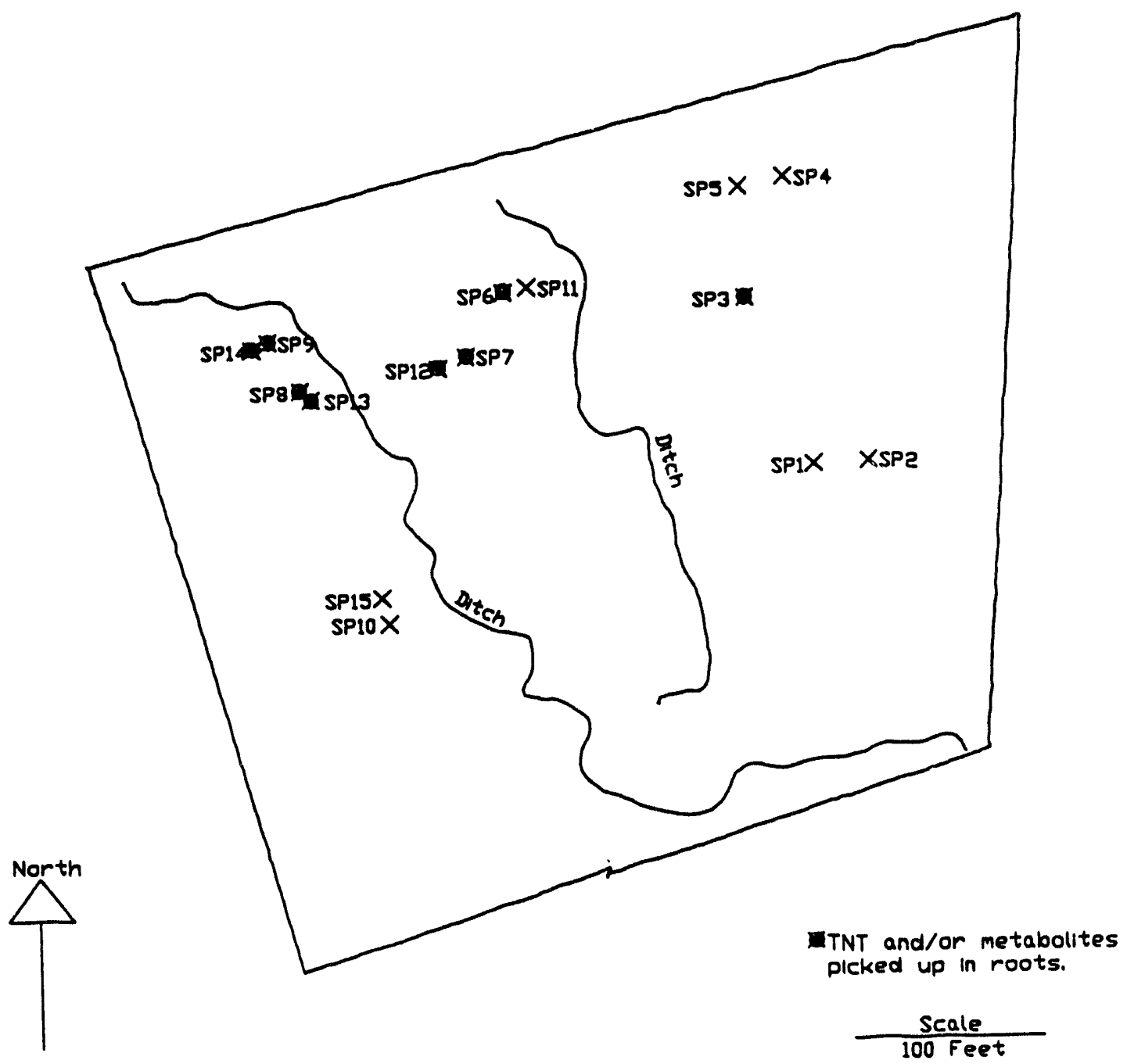

FIGURE 3 TNT Sample Locations at the Joliet Army Ammunition Plant, Group 61 - Ridge and Furrow Area

3. A $2.5-\mathrm{mL}$ aliquot of dichlorometharie extract is removed, placed in a glass scintillation vial, and evaporated to dryness by air.

4. Five milliliters of $15 \%$ hexane in dichloromethane is added on the surface of a Florisil solid-phase extraction cartridge to equilibrate the cartridge.

5. The residue of sample from Step 3 is dissolved in $1 \mathrm{~mL}$ of $15 \%$ hexane in dichloromethane and added on the surface of the cartridge.

6. Three milliliters of $15 \%$ hexane in dichloromethane is eluted through the Florisil cartridge to wash out the less polar compounds. 
TABLE 1 Results of Explosives Analysisa

\begin{tabular}{|c|c|c|c|c|c|c|c|c|c|}
\hline \multirow[b]{2}{*}{ Location } & \multicolumn{2}{|r|}{ Plant } & \multirow[b]{2}{*}{ Soil TNT } & \multicolumn{3}{|c|}{ Plant Trp } & \multicolumn{3}{|c|}{ Root } \\
\hline & Common Name & Botanical Name & & TNT & 4-ADNT & 2-ADNT & TNT & 4-ADNT & 2-ADNT \\
\hline $\begin{array}{l}\text { SP1 } \\
\text { SP1 } \\
\text { SP2 } \\
\text { SP2 } \\
\text { SP3 } \\
\text { SP3 } \\
\text { SP3 } \\
\text { SP4 } \\
\text { SP4 } \\
\text { SP5 } \\
\text { SP5 } \\
\text { SP6 } \\
\text { SP6 } \\
\text { SP7 } \\
\text { SP7 } \\
\text { SP8 } \\
\text { SP8 } \\
\text { SP9 } \\
\text { SP9 } \\
\text { SP10 } \\
\text { SP10 } \\
\text { SP11 } \\
\text { SP12 } \\
\text { SP12 } \\
\text { SP13 } \\
\text { SP13 } \\
\text { SP14 } \\
\text { SP14 } \\
\text { SP14 } \\
\text { SP14 } \\
\text { SP15 } \\
\text { SP15 }\end{array}$ & $\begin{array}{l}\text { Bromegrass } \\
\text { Milkweed } \\
\text { Bromegrass } \\
\text { Teasel } \\
\text { False Boneset } \\
\text { Milkweed } \\
\text { Bromegrass } \\
\text { Vervain } \\
\text { Bromegrass } \\
\text { Ground Cherry } \\
\text { Bromegrass } \\
\text { Teasel } \\
\text { Bromegrass } \\
\text { Teasel } \\
\text { Bromegrass } \\
\text { Milkweed } \\
\text { Bromegrass } \\
\text { Bromegrass } \\
\text { Alfalfa } \\
\text { Chicory } \\
\text { Queen Anne's Lace } \\
\text { Bromegrass } \\
\text { Teasel } \\
\text { Bromegrass } \\
\text { Alfalia } \\
\text { Bromegrass } \\
\text { Milkweed } \\
\text { Chicory } \\
\text { Bromegrass } \\
\text { Alfalfa } \\
\text { Chicory } \\
\text { Queen Anne's Lace }\end{array}$ & $\begin{array}{l}\text { Bromus inermis } \\
\text { Asclepias syriaca } \\
\text { Bromus inermis } \\
\text { Dipsacus sylvestris } \\
\text { Kuhnia eupatorioides } \\
\text { Asclepias syriaca } \\
\text { Bromus inermis } \\
\text { Verbena hastata } \\
\text { Bromus inermis } \\
\text { Physalis heterophylla } \\
\text { Bromus inermis } \\
\text { Dipsacus sylvestris } \\
\text { Bromus inermis } \\
\text { Dipsacus sylvestris } \\
\text { Bromus inermis } \\
\text { Asclepias syriaca } \\
\text { Bromus inermis } \\
\text { Bromus inermis } \\
\text { Medicago sativa } \\
\text { Cichorium intybus } \\
\text { Daucus carota } \\
\text { Bromus inermis } \\
\text { Dipsacus sylvestris } \\
\text { Bromus inermis } \\
\text { Medicago sativa } \\
\text { Bromus inermis } \\
\text { Asclepias syriaca } \\
\text { Cichorium intybus } \\
\text { Bromus inermis } \\
\text { Medicago saiiva } \\
\text { Cichorium intybus } \\
\text { Daucus carota } \\
\text { DETECTION LiMIT }\end{array}$ & $\begin{array}{c}B^{B D L^{b}} \\
B D L \\
B D L \\
B D L \\
1.00 \\
1.00 \\
1.00 \\
1.50 \\
1.50 \\
1.60 \\
1.60 \\
6,260 \\
6,260 \\
492 \\
492 \\
278 \\
278 \\
5,840 \\
5,840 \\
3,360 \\
3,360 \\
3,410 \\
39,350 \\
39,350 \\
5,340 \\
5,340 \\
3,350 \\
3,350 \\
3,350 \\
3,350 \\
202 \\
202\end{array}$ & $\begin{array}{l}\mathrm{BDL} \\
\mathrm{BDL} \\
\mathrm{BDL} \\
\mathrm{BDL} \\
\mathrm{BDL} \\
\mathrm{BDL} \\
\mathrm{BDL} \\
\mathrm{BDL} \\
\mathrm{BDL} \\
\mathrm{BDL} \\
\mathrm{BDL} \\
\mathrm{BDL} \\
\mathrm{BDL} \\
\mathrm{BDL} \\
\mathrm{BDL} \\
\mathrm{BDL} \\
\mathrm{BDL} \\
\mathrm{BDL} \\
\mathrm{BDL} \\
\mathrm{BDL} \\
\mathrm{BDL} \\
\mathrm{BDL} \\
\mathrm{BDL} \\
\mathrm{BDL} \\
\mathrm{BDL} \\
\mathrm{BDL} \\
\mathrm{BDL} \\
\mathrm{BDL} \\
\mathrm{BDL} \\
\mathrm{BDL} \\
\mathrm{BDL} \\
\mathrm{BDL} \\
0.08\end{array}$ & $\begin{array}{l}\mathrm{BDL} \\
\mathrm{BDL} \\
\mathrm{BDL} \\
\mathrm{BDL} \\
\mathrm{BDL} \\
\mathrm{BDL} \\
\mathrm{BDL} \\
\mathrm{BDL} \\
\mathrm{BDL} \\
\mathrm{BDL} \\
\mathrm{BDL} \\
\mathrm{BDL} \\
\mathrm{BDL} \\
\mathrm{BDL} \\
\mathrm{BDL} \\
\mathrm{BDL} \\
\mathrm{BDL} \\
\mathrm{BDL} \\
\mathrm{BDL} \\
\mathrm{BDL} \\
\mathrm{BDL} \\
\mathrm{BDL} \\
\mathrm{BDL} \\
\mathrm{BDL} \\
\mathrm{BDL} \\
\mathrm{BDL} \\
\mathrm{BDL} \\
\mathrm{BDL} \\
\mathrm{BDL} \\
\mathrm{BDL} \\
\mathrm{BDL} \\
\mathrm{BDL} \\
0.20\end{array}$ & $\begin{array}{l}\mathrm{BDL} \\
\mathrm{BDL} \\
\mathrm{BDL} \\
\mathrm{BDL} \\
\mathrm{BDL} \\
\mathrm{BDL} \\
\mathrm{BDL} \\
\mathrm{BDL} \\
\mathrm{BDL} \\
\mathrm{BDL} \\
\mathrm{BDL} \\
\mathrm{BDL} \\
\mathrm{BDL} \\
\mathrm{BDL} \\
\mathrm{BDL} \\
\mathrm{BDL} \\
\mathrm{BDL} \\
\mathrm{BDL} \\
\mathrm{BDL} \\
\mathrm{BDL} \\
\mathrm{BDL} \\
\mathrm{BDL} \\
\mathrm{BDL} \\
\mathrm{BDL} \\
\mathrm{BDL} \\
\mathrm{BDL} \\
\mathrm{BDL} \\
\mathrm{BDL} \\
\mathrm{BDL} \\
\mathrm{BDL} \\
\mathrm{BDL} \\
\mathrm{BDL} \\
0.20\end{array}$ & $\begin{array}{l}\mathrm{BDL} \\
\mathrm{BDL} \\
\mathrm{BDL} \\
\mathrm{BDL} \\
0.13 \\
0.60 \\
4.50 \\
\mathrm{BDL} \\
\mathrm{BDL} \\
\mathrm{BDL} \\
\mathrm{BDL} \\
0.15 \\
2.33 \\
\mathrm{BDL} \\
\mathrm{BDL} \\
\mathrm{BDL} \\
0.29 \\
5.85 \\
\mathrm{NT} \\
\mathrm{BDL} \\
\mathrm{BDL} \\
\mathrm{BDL} \\
\mathrm{BDL} \\
3.85 \\
\mathrm{BDL} \\
0.86 \\
\mathrm{BDL} \\
\mathrm{BDL} \\
\mathrm{BDL} \\
\mathrm{BDL} \\
\mathrm{BDL} \\
\mathrm{BDL} \\
0.08\end{array}$ & $\begin{array}{l}\mathrm{BDL} \\
\mathrm{BDL} \\
\mathrm{BDL} \\
\mathrm{BDL} \\
\mathrm{BDL} \\
\mathrm{BDL} \\
\mathrm{BDL} \\
\mathrm{BDL} \\
\mathrm{BDL} \\
\mathrm{BDL} \\
\mathrm{BDL} \\
2.12 \\
4.29 \\
0.57 \\
0.63 \\
\mathrm{BDL} \\
0.88 \\
5.71 \\
\mathrm{NT} \\
\mathrm{BDL} \\
\mathrm{BDL} \\
\mathrm{BDL} \\
\mathrm{BDL} \\
3.72 \\
\mathrm{BDL} \\
2.74 \\
\mathrm{BDL} \\
\mathrm{BDL} \\
1.55 \\
\mathrm{BDL} \\
\mathrm{BDL} \\
\mathrm{BDL} \\
0.20\end{array}$ & $\begin{array}{l}\text { BDL } \\
B D L \\
B D L \\
B D L \\
0.93 \\
B D L \\
B D L \\
B D L \\
B D L \\
B D L \\
B D L \\
1.15 \\
4.43 \\
B D L \\
B D L \\
B D L \\
1.28 \\
7.71 \\
\text { NT } \\
B D L \\
B D L \\
B D L \\
B D L \\
4.35 \\
B D L \\
3.20 \\
B D L \\
B D L \\
1.13 \\
B D L \\
B D L \\
B D L \\
0.20\end{array}$ \\
\hline
\end{tabular}

a All units $\mathrm{mg} / \mathrm{kg}$ (ppm).

b $\mathrm{BDL}=$ Below Detection Limit. 
7. Five milliliters of $12 \%$ ethyl acetate in dichloromethane is added to elute the explosive compounds.

8. The collected fraction is evaporated to dryness, and the residue is dissolved in $1 \mathrm{~mL}$ of acetonitrile. One milliliter of HPLC-grade water is added, and the sample mixture is filtered through a Nalgene $0.2-\mu \mathrm{m}$ filter. The sample is then analyzed by HPLC.

These analyses were performed in a USATHAMA-certified laboratory at the University of Illinois at Urbana, Illinois. Spiked samples were run to check the validity of the procedure. On plant tops, spike recovery averaged $89 \%$ for TNT, $60 \%$ for $4-\mathrm{ADNT}$, and $90 \%$ for 2 -ADNT. For roots, the spike recovery averaged $66 \%$ for TNT, $48 \%$ for $4-\mathrm{ADNT}$, and $84 \%$ for 2 -ADNT.

Four soil samples for TCLP analyses were collected from each quadrant of Group 61 (Figure 4). Samples were placed in precleaned glass bottles, sealed, and delivered to a commercial laboratory (Heritage Laboratories, Inc.) for TCLP analysis by means of EPA method SW846-1311. Results from the TCLP analyses were used to characterize the toxicity of the soil from the ridge-and-furrow area.

Table 2 contains the analytical results of the TCLP samples taken in the ridge-and-furrow area of Grolip 61. No targeted TCLP compounds were detected in the samples. For the purposes of this study, the results indicate that the soil in Group 61 cannot be classified as a toxic waste. 


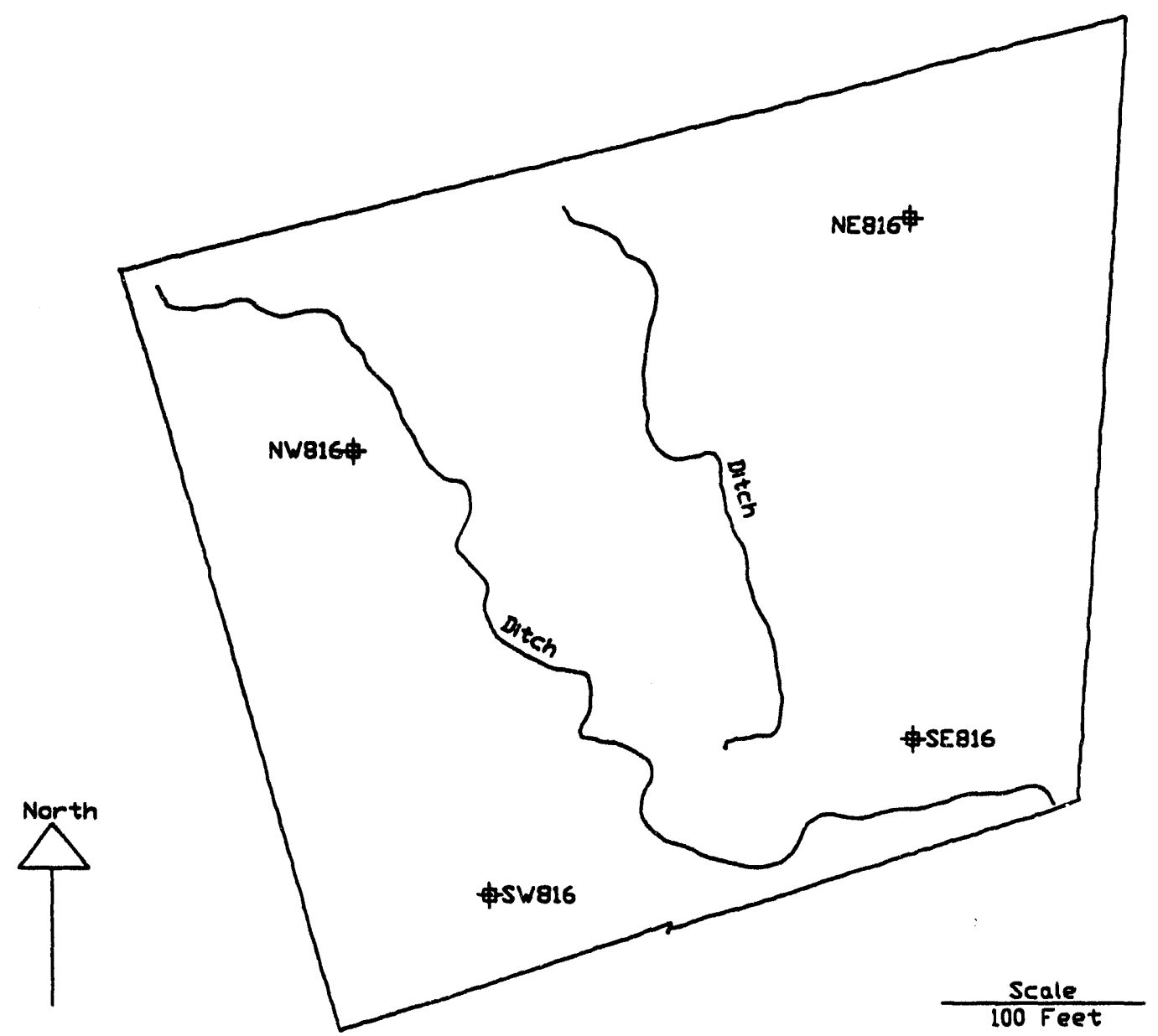

FIGURE 4 TCLP Sample Locations at the Joliet Army Ammunition Plant, Group 61 Ridge and Furrow Area 


\begin{tabular}{|c|c|c|c|c|c|}
\hline \multirow[b]{2}{*}{ Analyte } & \multicolumn{4}{|c|}{ Sample } & \multirow{2}{*}{$\begin{array}{l}\text { Detection } \\
\text { Limits }\end{array}$} \\
\hline & MM & NE & sw & $S E$ & \\
\hline $\begin{array}{l}\text { Barium } \\
\text { Cadmium }\end{array}$ & $\begin{array}{c}3.4 \\
B D L^{b}\end{array}$ & $\begin{array}{l}1 \\
\text { BDL }\end{array}$ & $\begin{array}{l}0.56 \\
B D L\end{array}$ & $\begin{array}{l}0.98 \\
B D L\end{array}$ & $\begin{array}{l}0.02 \\
0.04\end{array}$ \\
\hline Chromium & BDL & BDL & BDL & BDL & 0.05 \\
\hline Lead & BDL & BDL & $\mathrm{BDL}$ & BDL & 0.25 \\
\hline Silver & BDL & BDL & $B D L$ & $B D L$ & 0.05 \\
\hline Arsenic & BDL & $\mathrm{BDL}$ & BDL & BDL & 0.01 \\
\hline Selenium & $B D L$ & $B D L$ & BDL & $B D L$ & 0.01 \\
\hline Mercury & $\mathrm{BDL}$ & BDL & BDL & BDL & 0.005 \\
\hline 1,4-Dichlorobenzene & $\mathrm{BDL}$ & BDL & BDL & BDL & 0.05 \\
\hline 2,4-Dinitrotoluene & $B D L$ & BDL & $\mathrm{BDL}$ & BDL & 0.05 \\
\hline Hexachlorobenzene & $\mathrm{BDL}$ & BDL & BDL & BDL & 0.05 \\
\hline Hexachlorobutadiene & BDL & BDL & BDL & $B D L$ & 0.05 \\
\hline Hexachloroethane & $\mathrm{BDL}$ & $\mathrm{BDL}$ & BDL & BDL & 0.05 \\
\hline Nitrobenzene & BDL & BDL & BDL & BDL & 0.05 \\
\hline Pyridine & BDL & $B D L$ & BDL & BDL & 0.25 \\
\hline 2-Methyl Phenol & BDL & BDL & BDL & $B D L$ & 0.13 \\
\hline 3-Methyl Phenol & $\mathrm{BDL}$ & BDL & BDL & BDL & 0.13 \\
\hline 4-Methyl Phenol & BDL & BDL & $\mathrm{BDL}$ & BDL & 0.13 \\
\hline Pentachlorophenol & $\mathrm{BDL}$ & $\mathrm{BDL}$ & BDL & $\mathrm{BDL}$ & 0.25 \\
\hline 2,4,5-Trichlorophenol & $\mathrm{BDL}$ & BDL & BDL & $B D L$ & 0.13 \\
\hline 2,4,6-Trichlorophenol & BDL & BDL & BDL & $\mathrm{BDL}$ & 0.13 \\
\hline Benzene & BDL & BDL & BDL & BDL & 0.05 \\
\hline Carbon Tetrachloride & $\mathrm{BDL}$ & BDL & BDL & BDL & 0.05 \\
\hline Chlorobenzene & BDL & BDL & BDL & BDL & 0.05 \\
\hline Chloroform & BDL & $B D L$ & $\mathrm{BDL}$ & BDL & 0.05 \\
\hline 1,2-Dichloroethane & $B D L$ & BDL & $B D L$ & BDL & 0.05 \\
\hline 1,1-Dichloroethylene & BDL & BDL & $\mathrm{BDL}$ & BDL & 0.05 \\
\hline Methyl Ethyl Ketone & $B D L$ & BDL & BDL & BDL & 0.1 \\
\hline Tetrachloroethylene & BDL & BDL & BDL & $B D L$ & 0.05 \\
\hline Trichloroethylene & BDL & $B D L$ & BDL & $\mathrm{BDL}$ & 0.05 \\
\hline Vinyl Chloride & $\mathrm{BDL}$ & $\mathrm{BDL}$ & BDL & $\mathrm{BDL}$ & 0.1 \\
\hline Lindane & BDL & $\mathrm{BDL}$ & BDL & BDL & \\
\hline Heptachlor & BDL & $B D L$ & $B D L$ & $B D L$ & \\
\hline Heptachlor Epoxide & $\mathrm{BDL}$ & $B D L$ & $\mathrm{BDL}$ & BDL & \\
\hline Endrin & BDL & BDL. & BDL & BDL & \\
\hline Methoxychlor & $B D L$ & $B D L$ & BDL & BDL & \\
\hline Chlordane & BDL & BDL & $\mathrm{BDL}$ & BDL & \\
\hline
\end{tabular}

all units $\mathrm{mg} / \mathrm{kg}$ (ppm).

b $\mathrm{BDL}=$ Below Detection Limit. 


\section{Results and Conclusions}

Table 1 (page 8) contains the analytical results for the soil and plant material sampled in 15 locations in the ridge-and-furrow area. Locations SP1 to SP5 were areas of low contamination (less than $5 \mathrm{mg} / \mathrm{kg}$ ). Locations SP6 to SP15 were areas of intermediate to high levels of TNT contamination. For all areas, only one sample (SP8 milkweed) gave an indication of TNT in the aboveground portion of the plant, and that was below the detection limit of $0.08 \mathrm{mg} / \mathrm{kg}$. Therefore, no detection of TNT or the amino compounds was confirmed in any of the plant parts above the ground. Some chromatograms contained peaks that represent unidentified organic compounds extracted from plants, but retention times did not match those for TNT or the amino derivatives. Figure 5 is a chromatogram of a standard mix of explosives.

The results indicate that TNT, 2-ADNT, and/or 4-ADNT were found in some root samples of false boneset (Kuhnia eupatorioides), teasel (Dipsacus sylvestris), and bromegrass (Bromus inermis). Visual observation of the root samples showed that the mechanical elutriator appeared to have removed all of the potential contaminants from the soils. However, it is possible that some slight soil contamination remained, especially in the case of the very fine roots for species like bromegrass, for which washing was more difficult. The presence of 2-ADNT and 4-ADNT, which could be plant metabolites of TNT, increases the likelihood that explosives were taken up by plant roots, as opposed to their presence resulting from external soil contamination. 


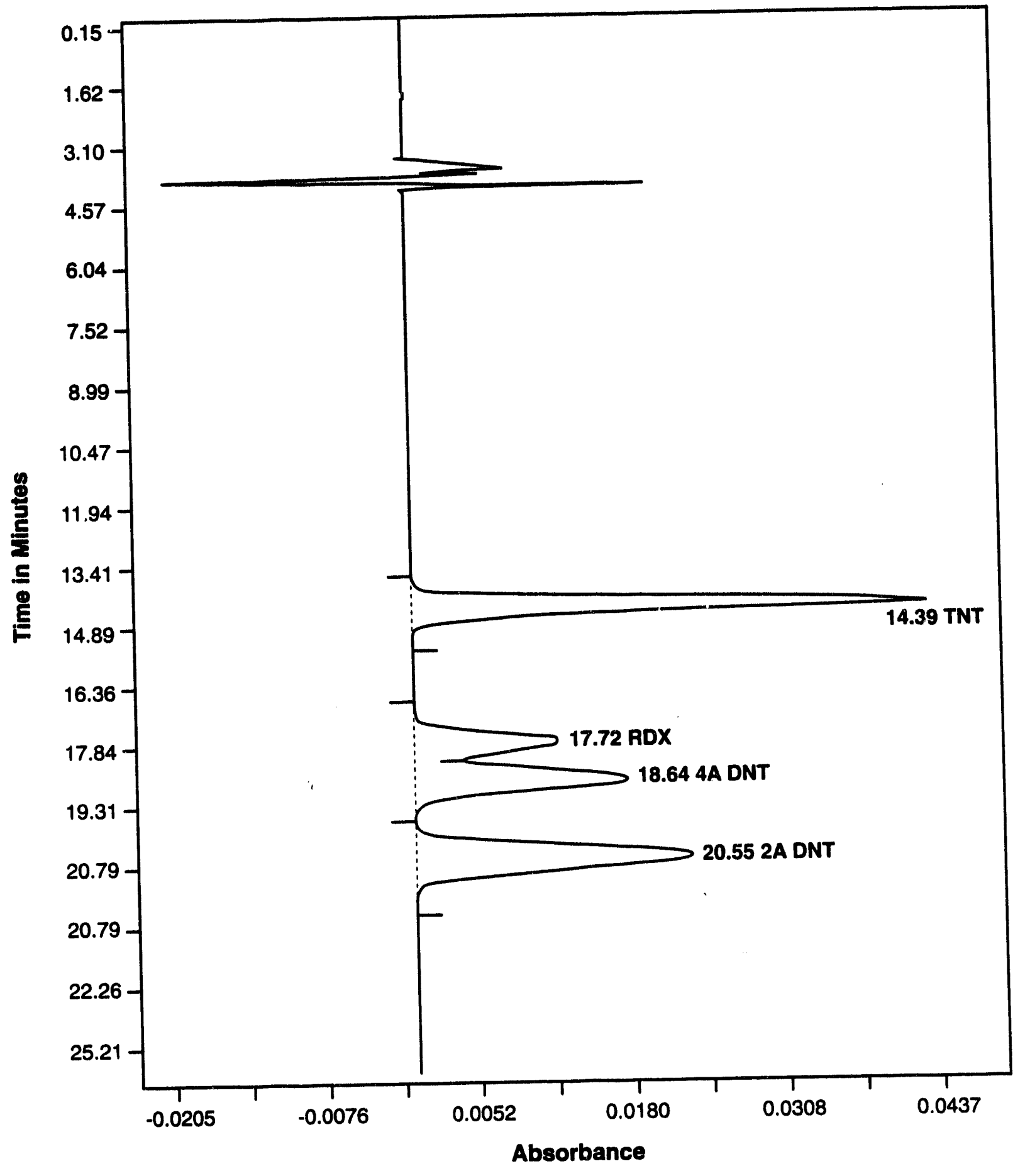

FIGURE 5 Chromatogram of Standard Explosives Mix 


\section{References}

Banwart, W.L., and J.J. Hassett, 1990, Effects of Soil Amendments on Plant Tolerance and Extractable TNT from TNT Contaminated Soils, in Agronomy Abstracts, American Society of Agronomy, Madison, Wis., p. 33.

Banwart, W.L., et al., 1991, Plant Uptake of RDX from Spiked Soils, in Agronomy Abstracts, American Society of Agronomy, Madison, Wis., p. 35.

Dames \& Moore, 1991, Final Phase I Technical Plan, Remedial Investigation/Feasibility' Study Load-Assemble-Package (LAP) Area, Joliet Army Ammunition Plant, Joliet, Illinois, prepared for U.S. Army Toxic and Hazardous Materials Agency, Aberdeen Proving Ground, Md., March 6.

Folsom, B.L. Jr., et al., 1988, Effects of Soil pH and Treatment Level on Persistence and Plant Uptake of 2,4,6-Trinitrotoluene, 1988 Final Report, EL-88-22, Environmental Laboratory, Waterways Experiment Station, U.S. Army Corps of Engineers, Vicksburg, Miss.

Harvey, S.D., et al., 1990, Analysis of 2,4,6-Trinitrotoluene and Its Transformation Products in Soils and Plant Tissues by High-Performance Liquid Chromatography, Journal of Chromatography, 518:361-374.

Harvey, S.D., et al., 1991, Fate of the Explosive Hexahydro-1,3,5-Trinitro-1,3 5-Triazine (Rdx) in Soil and Bioaccumulation in Bush Bean Hydroponic Plants, Environmental Toxicology and Chemistry, 10:845-855.

Harvey, S.D., et al., 1993, Analysis of the Explosive 2,4,6-trinitrophenylmethylnitramine (tetryl) in Bush Bean Plants, Journal of Chromatography, 630:167-177.

Palazzo, A.J., and D.C. Leggett, 1986, Effect and Disposition of TNT in a Terrestrial Plant, Journal of Environmental Quality, 15:49

Pennington, J.C., 1988, Soil Sorption and Plant Uptake of 2,4,6-Trinitrotoluene, Final Report, EL-88-12, Environmental Laboratory, Waterways Experiment Station, U.S. Army Corps of Engineers, Vicksburg, Miss.

Ritchie, E.S., et al., 1991, Phase 1 Remedial Investigation/ Feasibility Study, Load-AssemblePackage (LAP) Area, Joliet Army Ammunition Plant, Joliet, Illinois, prepared by Dames \& Moore, Bethesda, Md., for U.S. Army Toxic and Hazardous Materials Agency, Aberdeen Proving Ground, Md., March.

U.S. Army Toxic and Hazardous Materials Agency, 1990, Quality Assurance Program, USATHAMA PAM 11-41, Aberdeen Proving Ground, Md. 


\section{Appendix A:}

\section{Analytical Chemistry Data from Previous Sampling Events at Group 61*}

* Adapted from Dames \& Moore 1991. (Samples SC091, SC092, and SC093 are from the ridge-and-furrow area.) 
Chemical Analysis Results from Group 61

\begin{tabular}{|c|c|c|c|c|c|c|c|c|c|c|c|c|c|}
\hline $\begin{array}{l}\text { Sample } \\
\text { Location| }\end{array}$ & Date & $\begin{array}{l}\text { Sample } \\
\text { Depth (cm) } \\
\text { or Aquiferl }\end{array}$ & Units & 2,4,6-TNT & 2,6-DNT & 2,4-DNT| & 2NT & |1,3,5-TNB & 1,3-DNB & TETRY & Nom | & $\mathbf{P D X}$ & the \\
\hline \multirow[t]{3}{*}{ Sc091 } & $5 / 27 / 81$ & 0 & $\mathrm{mg} / \mathrm{kg}$ & $14,400.00+$ & $B D$ & BD & $\mathrm{BO}$ & $235.00+$ & - & - & - & - & - \\
\hline & $5 / 27 / 81$ & 15 & $\mathrm{mg} / \mathrm{kg}$ & \begin{tabular}{|l|}
$25.00+$ \\
\end{tabular} & $\mathrm{BD}$ & $\mathrm{BOL}$ & BOL & $10.20+$ & - & $=$ & - & - & - \\
\hline & $5 / 27 / 81$ & 30 & $\mathrm{mg} / \mathrm{kg}$ & $5.42+$ & $B O L$ & $B D$ & $E D L$ & $B D$ & - & - & - & - & - \\
\hline \multirow[t]{3}{*}{ Sc092 } & $5 / 26 / 81$ & 0 & $\mathrm{mg} / \mathrm{kg}$ & $20.30+$ & $\mathrm{BOL}$ & $B D$ & $\mathrm{BD}$ & BOL & - & - & - & - & - \\
\hline & $5 / 26 / 81$ & 18 & $\mathrm{mg} / \mathrm{kg}$ & $3.14+$ & BDL & $B D$ & $\mathbf{E D}$ & EDL & - & - & - & - & - \\
\hline & $5 / 26 / 81$ & 43 & $\mathrm{mg} / \mathrm{kg}$ & $20.00+$ & $\mathrm{BD}$ & $\mathbf{B O}$ & BOL & BOL & - & - & - & - & - \\
\hline \multirow[t]{3}{*}{ sco93 } & $5 / 26 / 81$ & 0 & $\mathrm{mg} / \mathrm{kg}$ & $14,500.00+$ & $B D$ & $12.70+$ & 10.4 & $182.00+$ & - & - & - & - & - \\
\hline & $5 / 26 / 81$ & 18 & $\mathrm{mg} / \mathrm{kg}$ & $6.27+$ & $\mathrm{BOL}$ & $\mathrm{BD}$. & BD & $28.90+$ & - & - & - & - & - \\
\hline & $5 / 26 / 81$ & 49 & $\mathrm{mg} / \mathrm{kg}$ & $430.00+$ & $B D L$ & $B D$ & BDL & $54.10+$ & - & - & - & - & - \\
\hline AC281 & $11 / 2 / 82$ & 0 & $\mathrm{mg} / \mathrm{kg}$ & $B D$ & $B D$ & $\mathrm{BD}$ & $\mathrm{BD}$ & $\mathrm{BDL}$ & $\div$ & - & - & - & - \\
\hline AC283 & $11 / 2 / 82$ & 0 & $\mathrm{mg} / \mathrm{kg}$ & $B D$ & $B D$ & $\mathrm{BDL}$ & BDL & BOL & - & - & - & - & - \\
\hline \multirow[t]{3}{*}{ SC285 } & $11 / 2 / 82$ & 0 & $\mathrm{mg} / \mathrm{kg}$ & $B D$ & $B D$ & $B D$ & $\mathrm{BDL}$ & $\mathrm{BD}$ & - & - & - & - & - \\
\hline & $11 / 2 / 82$ & 30 & $\mathrm{mg} / \mathrm{kg}$ & $\mathrm{BDL}$ & $\mathrm{BDC}$ & BOL & $\mathrm{BDL}$ & $\mathbf{B D}$ & - & - & - & - & - \\
\hline & $11 / 2 / 82$ & 61 & $\mathrm{mg} / \mathrm{kg}$ & $B D$ & $\mathrm{BD}$ & $\mathrm{BD}$ & BOL & $B D$ & - & - & - & - & $\cdot$ \\
\hline \multirow[t]{4}{*}{ MW131 } & $6 / 10 / 81$ & $\underline{u}$ & $\mathrm{mg} / \mathrm{kg}$ & $2,250.00+$ & $3.40+$ & $0.98+$ & BDL & $973.00+$ & $\therefore$ & - & - & - & $\cdot$ \\
\hline & $6 / 24 / 82 \mathrm{~A}$ & U & $\mathrm{mg} / \mathrm{kg}$ & $744.00+$ & 1.80 & $0.70+$ & $1.90+$ & $725.00+$ & - & $\dot{-}$ & - & - & - \\
\hline & $11 / 15 / 85$ & $u$ & $\mathrm{mg} / \mathrm{kg}$ & $2,150.00+$ & $4.14+$ & $2.01+$ & - & $1,610.00+$ & $5.00+$ & $58.60+$ & $\therefore$ & $\mathrm{BOL}$ & - \\
\hline & $4 / 22 / 84$ & $\underline{u}$ & $\mathrm{mg} / \mathrm{kg}$ & $576.00+$ & $8.54+$ & $\mathrm{BD}$ & - & $755.00+$ & $\mathrm{BD}$ & 21.7 & - & $\mathrm{BO}$ & - \\
\hline \multirow[t]{3}{*}{ MW172 } & $3 / 9 / 83$ & D & $\mathrm{mg} / \mathrm{kg}$ & $40.80+$ & $\mathrm{BDL}$ & $\mathrm{BD}$ & $B D$ & $9.20+$ & - & $\cdot$ & $\therefore$ & - & $\therefore$ \\
\hline & $10 / 30 / 85$ & D & $\mathrm{mg} / \mathrm{kg}$ & $16.20+$ & $\mathrm{BD}$ & $\mathrm{BOL}$ & - & $3.06+$ & 80 & $\mathrm{BOL}$ & 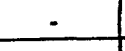 & $14.20+$ & $\cdot$ \\
\hline & $4 / 14 / 86$ & D & $\mathrm{mg} / \mathrm{kg}$ & $12.90+$ & $\mathrm{BOL}$ & $B D$ & $\div$ & $3.84+$ & $\mathrm{BD}$ & $B D$ & - & 7.22 & - \\
\hline \multirow[t]{3}{*}{ MW173 } & $3 / 9 / 83$ & $\mathbf{u}$ & $\mathrm{mg} / \mathrm{kg}$ & $50.30+$ & $\mathrm{BO}$ & $\mathrm{BOL}$ & $B D$ & $6.87+$ & - & - & - & - & $=$ \\
\hline & $10 / 31 / 85$ & $u$ & $\mathrm{mg} / \mathrm{kg}$ & $105.00+$ & $\mathrm{BOL}$ & $\mathrm{BOL}$ & $\cdot$ & $14.00+$ & $\mathrm{BOL}$ & $\mathrm{BOL}$ & - & $56.50+$ & - \\
\hline & $4 / 14 / 86$ & $\underline{u}$ & $\mathrm{mg} / \mathrm{kg}$ & $11.00+$ & $\mathrm{BD}$ & $\mathrm{BOL}$ & - & $2.09+$ & $\mathrm{BDL}$ & $B D L$ & - & 8 & - \\
\hline \multirow[t]{3}{*}{ MW174 } & $3 / 10 / 83$ & $u$ & $\mathrm{mg} / \mathrm{kg}$ & $\mathrm{BOL}$ & $B D$ & $\mathrm{BO}$ & $\mathrm{BD}$ & $B O L$ & - & - & - & - & - \\
\hline & $10 / 31 / 85$ & $\underline{u}$ & $\mathrm{mg} / \mathrm{kg}$ & $\mathrm{BO}$ & $8 D$ & $B D$ & - & $B D$ & $\mathrm{BDL}$ & $B D$ & - & $B D$ & - \\
\hline & $4 / 14 / 86$ & $\underline{u}$ & $\mathrm{mg} / \mathrm{kg}$ & $B O L$ & $B Q$ & $\mathrm{BOL}$ & - & $B D$ & $\mathrm{BDL}$ & $\mathrm{BO}$ & - & $B D$ & $\cdot$ \\
\hline MW175 & $4 / 14 / 86$ & $\mathbf{U}$ & $\mathrm{mg} / \mathrm{kg}$ & $\mathrm{BOL}$ & $\mathrm{BO}$ & $\mathrm{BD}$ & - & $B D$ & $B D$ & $\mathrm{BDL}$ & - & $\mathrm{BOL}$ & - \\
\hline \multirow[t]{3}{*}{ MW177 } & $3 / 9 / 83$ & $u$ & $\mathrm{mg} / \mathrm{kg}$ & 0.31 & $B D$ & $\mathrm{BD}$ & $\mathrm{BDL}$ & $\mathrm{BOL}$ & - & - & - & - & - \\
\hline & $10 / 30 / 85$ & $U$ & $\mathrm{mg} / \mathrm{kg}$ & $\mathrm{BOL}$ & $\mathrm{BD}$ & $B D$ & - & $B O L$ & $\mathrm{BD}$ & $\mathrm{BD}$ & - & $B D$ & - \\
\hline & $4 / 14 / 86$ & $u$ & \begin{tabular}{|l|}
$\mathrm{mg} / \mathrm{kg}$ \\
\end{tabular} & $\mathrm{BDL}$ & $\mathrm{BD}$ & $\mathrm{BOL}$ & - & $B a$ & $\mathrm{BD}$ & $\mathrm{BOL}$ & - & $\mathrm{BO}$ & - \\
\hline \multirow[t]{3}{*}{ MW178 } & $3 / 9 / 83$ & $\underline{u}$ & $\mathrm{mg} / \mathrm{kg}$ & 0.38 & $B D$ & $\mathrm{BD}$ & $B D$ & $B D$ & - & - & - & - & $\cdot$ \\
\hline & $11 / 6 / 85$ & $u$ & $\mathrm{mg} / \mathrm{kg}$ & $\mathrm{BO}$ & $\mathrm{BOL}$ & $\mathrm{BDL}$ & - & $\mathrm{BD}$ & $\mathrm{BOL}$ & $\mathrm{BOL}$ & - & $\mathrm{BD}$ & - \\
\hline & $4 / 14 / 86$ & $\mathrm{U}$ & $\mathrm{mg} / \mathrm{kg}$ & $\mathrm{BOL}$ & $\mathrm{BDL}$ & $\mathrm{BO}$ & 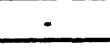 & $\mathrm{BOL}$ & $\mathrm{BD}$ & $\mathrm{BDL}$ & - & $\mathrm{BD}$ & - \\
\hline
\end{tabular}


Appendix B:

Summary of Analysis for Explosives from Previous Sampling Events at Group 61 


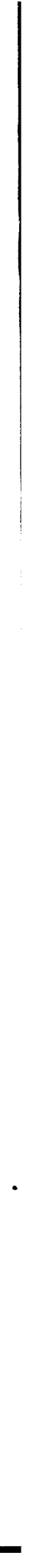

- 
SUMARY OF AMPLYSIS FOR EXPLOSIVES

SAMPLES RECEIVED FROM JOLIET APP

METHOO OF ANPLYSIS: EPA METHOD 8330: ACN EXTRACTION

\begin{tabular}{|c|c|c|c|c|c|c|c|c|c|c|c|c|c|c|c|c|c|}
\hline LOCATION & $\begin{array}{l}\text { SAMPLE } \\
\text { MUMEER }\end{array}$ & $\begin{array}{l}\text { DATE } \\
\text { REC'O }\end{array}$ & $\begin{array}{l}\text { OATE } \\
\text { EXIR }\end{array}$ & $\begin{array}{l}\text { DATE } \\
\text { RARR_YZEO }\end{array}$ & $\begin{array}{l}\text { DATE } \\
\text { CONFIRHED }\end{array}$ & nure. & $\operatorname{Imx}$ & TMS & $\mathbf{R I X X}$ & $\begin{array}{c}\text { CONCENTS } \\
\text { DWES }\end{array}$ & $\underset{\text { NG }}{\mathrm{ATION}}$ & III SOIL & $\begin{array}{l}\text { SAMPLES } \\
240 \mathrm{NT}\end{array}$ & $\begin{array}{l}\text { (ug/g) } \\
260 \mathrm{dt}\end{array}$ & pilt & 2ravt & Amont \\
\hline \multicolumn{18}{|c|}{ SOIL SAMPLES FROH JOLIET ARP } \\
\hline CLS1004m4-MS & ค-1 & & $12 / 18 / 91$ & $12 / 21 / 91$ & $12 / 22 / 91$ & PIm1 & $<0$ & 177 & 31.6 & 1.80 & $<0$ & 29800 & 19.2 & $\mathbf{< 0}$ & $\infty$ & $<0$ & $<0$ \\
\hline CLSIOOHM4-HS & A-2 & & $12 / 18 / 91$ & $12 / 21 / 91$ & $12 / 22 / 91$ & PIM & $<0$ & 171 & 28.2 & 2.70 & $<0$ & 31100 & 13.6 & $<0$ & 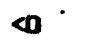 & $<0$ & $<0$ \\
\hline CLSIOOHWA-HS & $\theta-1$ & & $12 / 18 / 91$ & $12 / 21 / 91$ & $12 / 22 / 91$ & PIUY & $<0$ & 179 & 29.5 & 2.60 & $<0$ & 27000 & 10.4 & $<0$ & $\mathbf{Q}$ & $<0$ & $<0$ \\
\hline CLS100um4-HS & $B-2$ & & $12 / 18 / 91$ & $12 / 21 / 91$ & $12 / 22 / 91$ & Pits & $<0$ & 162 & 39.4 & 1.90 & $<0$ & 23600 & 9.80 & $\boldsymbol{\infty}$ & $\infty$ & $<0$ & $<0$ \\
\hline SOIL BLFN & & & $12 / 18 / 91$ & $12 / 21 / 91$ & $12 / 22 / 91$ & Pter & $<0$ & $<0$ & $<0$ & $<\mathbf{D}$ & $<3$ & $<0$ & $<0$ & $<0$ & $\boldsymbol{D}$ & $<$ & $<0$ \\
\hline \multirow[t]{4}{*}{ SPIKED SOIL } & A & & $12 / 18 / 91$ & $12 / 21 / 91$ & $12 / 22 / 91$ & PHI & 4.47 & 0.57 & 0.90 & 0.92 & & 0.73 & 0.99 & & & 0.76 & \\
\hline & 日 & & $12 / 18 / 91$ & $12 / 21 / 91$ & $12 / 22 / 91$ & PIM & 1.12 & 0.55 & n. 76 & 0.86 & & 0.73 & 0.99 & & & 0.75 & \\
\hline & MOHI COMC & & & & & & 5.00 & 1.00 & 1.00 & 1.00 & & 1.01 & 1.00 & & & 0.70 & \\
\hline & $\begin{array}{l}\text { RTIFIED RE } \\
=\text { TRACE LE }\end{array}$ & TING & $\begin{array}{l}\text { IHITS rug } \\
\text { CTED bur }\end{array}$ & $\begin{array}{l}\text { goNCENTRAT } \\
\text { CoN }\end{array}$ & $M$ & & ${ }_{C R L}^{2.20}$ & 0.24 & 1.00 & D. 12 & 0.11 & 0.24 & 0.07 & 0.16 & 0.25 & 0.11 & 0.2 ext \\
\hline
\end{tabular}


Appendix C:

Documentation of Analytical Method (LW-22) for Explosives Analysis 
DOCUMENTATION OF ANALYTICAL METHOD (LW-22)

FOR EXPLOSIVES ANALYSIS

\section{Summary}

A. Analytes
Chemical Name
Abbreviation
1. hexahydro - 1,3,5 - trinitro - 1,3,5 - triazine (RDX)
2. 1,3,5 - trinitrobenzene
(135TNB)
3. 2,4,6 - trinitrotoluene
(246TNT)
4. 2,6 - dinitrotoluene
(26DNT)
5. 2,4 - dinitrotoluene
(24DNT)

\section{B. Matrix}

The matrix is soil.

C. General Method for Explosives Analysis in Soils

Soil is extracted with acetonitrile. The extractant is combined with an aqueous $\mathrm{CaCl}_{2}$ solution and filtered. The filtered extract is analyzed by UV detection on reverse phase HPLC. Analyte concentrations in the soil are then calculated.

II. Application

A. Tested Concentration Range (soil matrix)

$\begin{array}{ll}\text { Analyte } & \text { Conc. range in a soil matrix } \\ \text { RDX } & 1.0 \mu \mathrm{g} / \mathrm{g} \text { to } 100 \mu \mathrm{g} / \mathrm{g} \text { soil } \\ \text { 135TNB } & 0.5 \mu \mathrm{g} / \mathrm{g} \text { to } 50 \mu \mathrm{g} / \mathrm{g} \text { soil } \\ \text { 246TNT } & 1.0 \mu \mathrm{g} / \mathrm{g} \text { to } 100 \mu \mathrm{g} / \mathrm{g} \text { soil } \\ \text { 26DNT } & 1.0 \mu \mathrm{g} / \mathrm{g} t 100 \mu \mathrm{g} / \mathrm{g} \text { soil } \\ \text { 24DNT } & 1.0 \mu \mathrm{g} / \mathrm{g} \text { to } 100 \mu \mathrm{g} / \mathrm{g} \text { soil }\end{array}$

B. Sensitivity

$\begin{array}{ll}\text { Analyte } & \\ \text { RDX } & 90.0 \text { Height Units for } 50 \mathrm{ng}=1.80 \mathrm{H} . \mathrm{U} . / \mathrm{ng} \\ \text { 135TNB } & 83.0 \text { Height Units for } 50 \mathrm{ng}=1.66 \mathrm{H} . \mathrm{U} . / \mathrm{ng} \\ \text { 246TNT } & 112.0 \text { Height Units for } 50 \mathrm{ng}=2.24 \mathrm{H} . \mathrm{U} . / \mathrm{ng} \\ \text { 26DNT } & 69.7 \text { Height Units for } 50 \mathrm{ng}=1.39 \mathrm{H} . \mathrm{U} . / \mathrm{ng} \\ \text { 24DNT } & 133.3 \mathrm{Height} \text { Units for } 50 \mathrm{ng}=2.67 \mathrm{H} . \mathrm{U} . / \mathrm{ng}\end{array}$


C. AEC Certified Reporting Limit ( $\mu \mathrm{g} / \mathrm{g})$

$\begin{array}{ll}\text { RDX } & 0.269 \\ \text { TNB } & 0.228 \\ \text { 26 DNT } & 0.569 \\ \text { 24 DNT } & 0.222 \\ \text { TNT } & 0.337\end{array}$

(See attached letter)

\section{Interferences}

If tetryl is present in environmental samples it may interfere with TNT analysis [Jenkins, Leggett, Grant, and Bauer, 1986]. However, it is not anticipated that tetryl will be present in high enougat concentrations to create a problem.

\section{E. Analysis Rate}

We estimate that 40 samples can be analyzed in an 8 hour day. The instrument in the laboratory is fully automated with an autosampler and data storage such that we have the capability of running analysis much longer than eight hours per day if necessary or desirable.

\section{F. Safety Information}

246TNT and RDX may be hazardous if absorbed through the skin or inhaled with particulate matter. 26DNT and 24DNT are considered to be toxic and 135TNB is considered to be a carcinogen. Care is taken to avoid skin contact with the compounds and to avoid inhalation of any particulate matter.

\section{Apparatus and Chemicals}

\section{A. Glassware and Hardware}

Gilson/Rainin pipetman \#F1000

Gilson/Rainin pipetman \#P200

Gilson/Rainin pipetman \#P2000

Volac 1-10 mL high precision dispenser \#MD049IX

$25 \mathrm{~mL}$ Corex glass screw top test tubes $\# 8446$

$20 \mathrm{~mL}$ screw top scintillation vials - Research Products Int'l Crop.

100 to $2000 \mathrm{~mL}$ glass graduated cylinders - Pyrex, Exax

0.1 to $10 \mathrm{~mL}$ glass pipettes - Coming, Kimax, Exax

10 to $100 \mathrm{~mL}$ glass volumetric flasks - Kimax, Pyrex 
5 to $200 \mu \mathrm{L}$. Eppendorf pipettes

Kimwipes wipers

50 to $2000 \mathrm{~mL}$ Erlenmeyer flasks - Kimax, Pyrex

20 to $2000 \mathrm{~mL}$ glass beakers - Kimax, Pyrex

various size plastic squeeze bottles

Amber glass jars, screw top

Sartorius balance \#1601A, max $110 \mathrm{~g}$

Mettler PE 3600 balance

B. Instrumentation

1. HPLC System

Beckman Analog Interface Module 406

Beckman Scanning Detector Module 167

Beckman model 110 - A Pump

Micromeritics Autoinjector Model 725

AST 286 Premium Computer

System Gold Chromatography Software

2. Sonic Probe

Sonics and Materials Inc.

Vibra Cell Sonic Probe Model VC250

3. Vortex

Curtin Matheson Scientific Inc.

Super Mixer Catalog Number 215-434

4. HPLC Parameters

Flow Rate

Mobile Phase

Length of Run

Sample Loop

Peak Width

Threshold

Wavelength
$1.5 \mathrm{~mL} / \mathrm{min}$

$58 \%$ methanol, $42 \%$ water

$10 \mathrm{~min}$

$50 \mu \mathrm{L}$

0.25

0.000150

$254 \mathrm{~nm}$ 


\section{Analytes}

1. Chemical Properties

$\begin{array}{lccll}\text { Analyte } & \begin{array}{l}\mathrm{mp} \\ \left({ }^{\circ} \mathrm{C}\right)\end{array} & \begin{array}{l}\text { bp } \\ \left({ }^{\circ} \mathrm{C}\right)\end{array} & \begin{array}{l}\text { density } \\ (\mathrm{g} / \mathrm{mL})\end{array} & \mathrm{CAS}-\# \\ \text { RDX } & 205 & - & 1.82 & 121-82-4 \\ \text { 135TNB } & 121 & 315 & 1.4775 & 25377-32-6 \\ \text { 246TNT } & 80 & 240 & 1.6540 & 118-96-7 \\ \text { 26DNT } & 66 & - & 1.2833 & 606-20-2 \\ \text { 24DNT } & 71 & 300 & 1.3207 & 121-14-2\end{array}$

2. Retention Times and Windows

\begin{tabular}{lcc}
\hline Compound & $\begin{array}{c}\text { Retention Time } \\
(\mathrm{min})\end{array}$ & $\begin{array}{c}\text { Window } \\
(\mathrm{min})\end{array}$ \\
RDX & 3.17 & 0.32 \\
135TNB & 4.16 & 0.42 \\
246TNT & 5.92 & 0.59 \\
26DNT & 6.54 & 0.65 \\
24DNT & 6.80 & 0.68
\end{tabular}

Note: Retention times may vary slightly as columns age or with slight variation in mobile phase solvent mixing.

\section{Reagents and SARMs}

1. HPLC grade Acetonitrile from Fisher Labs (99.9\%)

2. HPLC grade Methanol from Fisher Labs (99.9\%)

3. HPLC grade Water from Millipore System (approx. $18 \mathrm{megaohm} / \mathrm{cm}$ )

4. All analytes prepared from Standard Analytical Reference Materials (SARMs) came from the Picattiny Arsenal. 
IV. Calibration

\section{A. Preparation of Standards}

\section{Concentrated Stock Solutions}

An individual stock solution was prepared for each analyte by the following method. The target reporting limit (TRL) for RDX, 246TNT, 24DNT, and 26DNT is $1 \mu \mathrm{g} / \mathrm{g}$ soil and for 135TNB it is 0.5 $\mu \mathrm{g} / \mathrm{g}$ soil. The soil is extracted at a 10:1 ratio. Therefore, the concentration of explosive in the extract comparable to the TRL limit is $0.1 \mathrm{mg} / \mathrm{L}$ for RDX, 246TNT, 24DNT, and 26DNT, and $0.05 \mathrm{mg} / \mathrm{L}$ for 135TNB. An amount of analyte is weighed out so that the final stock solution has a concentration of $1000 *$ TRL (if the TRL is $0.1 \mathrm{mg} / \mathrm{L}$, then the stock solution is actually $100 \mathrm{mg} / \mathrm{L}$; if the TRL is $0.5 \mathrm{mg} / \mathrm{L}$, then the stock solution concentration is $50 \mathrm{mg} / \mathrm{L}$ ). The exact weights and volumes of the analyte and solution are listed on the next page.

\section{SARMs STOCK SOLUTIONS}

\begin{tabular}{lccc}
\hline Compound & $\begin{array}{c}\text { Weight } \\
(\mathrm{mg})\end{array}$ & $\begin{array}{c}\text { Volume } \\
(\mathrm{mL})\end{array}$ & $\begin{array}{c}\text { Conc. } \\
(\mathrm{mg} / \mathrm{L})\end{array}$ \\
RDX & 25.0 & 250 & 100.0 \\
135TNB & 12.5 & 250 & 50.0 \\
246TNT & 25.0 & 250 & 100.0 \\
26DNT & 25.0 & 250 & 100.0 \\
24DNT & 25.0 & 250 & 100.0
\end{tabular}

The solvent is HPLC grade acetonitrile (ACN). The SARMs stock sclutions are made in $250 \mathrm{~mL}$ volumetric flasks and are stored in the dark (wrapped flask in aluminum foil) in the freezer (about $-10^{\circ} \mathrm{C}$ ). They are checked periodically and are expected to have a shelf life of approximately 6 months.

\section{Working Stock Solutions}

A working stock solution (Working Stock I) was prepared with a concentration of approximately $10 \mathrm{mg} / \mathrm{L}$ of RDX, 246TNT, 26DNT, and 24DNT; $5 \mathrm{mg} / \mathrm{L}$ 135TNB. Working Stock I was prepared by adding 10.00 $\mathrm{mL}$ of each of the five stock solutions to a $100 \mathrm{~mL}$ volumetric flask with a volumetric pipet, diluting to volume with HPLC grade methanol, and thoroughly mixing. These working stock solutions were stored in the freezer and have a shelf life of 2 weeks. The preparation is shown in tabular form below. 


\section{WORKING STOCK I}

\begin{tabular}{|lccc|}
\hline Analyte & $\begin{array}{c}\text { Conc. in Stock } \\
(\mathrm{mg} / \mathrm{L})\end{array}$ & Dilution & $\begin{array}{c}\text { Concentration } \\
(\mathrm{mg} / \mathrm{L})\end{array}$ \\
\hline RDX & 100 & $1: 10$ & 10 \\
135TNB & 50 & $1: 10$ & 5 \\
246TNT & 100 & $1: 10$ & 10 \\
26DNT & 100 & $1: 10$ & 10 \\
24DNT & 100 & $1: 10$ & 10 \\
\hline
\end{tabular}

\section{Daily Calibration Standards}

The daily calibration standards are made by diluting an appropriate volume of the working stock solution. For example, if a daily standard of approximately $1.0 \mathrm{mg} / \mathrm{L}$ is desired, then $0.50 \mathrm{~mL}$ of working stock I (measured out with a glass pipet) is diluted to $5.00 \mathrm{~mL}$ with $4.50 \mathrm{~mL}$ methanol. The shelf life for the daily standards is one day. Preparation of each of the daily standards is shown below.

\section{DAILY CALIBRATION STANDARDS}

\begin{tabular}{|c|c|c|c|c|c|c|c|c|c|}
\hline \multirow{3}{*}{$\begin{array}{l}\operatorname{Tgt}^{1} \\
\text { Con. } \\
(\mu g / L)\end{array}$} & \multirow{2}{*}{$\begin{array}{l}\text { Soln } 2 \\
\text { Used }\end{array}$} & \multirow{2}{*}{\multicolumn{3}{|c|}{$\begin{array}{l}\text { Volume Added } \\
\text { Soln Dil. Total } \\
\text { Soln. }\end{array}$}} & \multicolumn{5}{|c|}{ Actual Concentration } \\
\hline & & & & & RDX & $\begin{array}{l}\text { 135- } \\
\text { TNB }\end{array}$ & $\begin{array}{l}\text { 246- } \\
\text { TNT }\end{array}$ & $\begin{array}{l}\text { 26- } \\
\text { DNT }\end{array}$ & $\begin{array}{l}\text { 24- } \\
\text { DNT }\end{array}$ \\
\hline & $\mathrm{ACN}$ & 0 & 5.00 & 5.00 & 0 & 0 & 0 & 0 & \\
\hline 100 & 1000 std. & 0.50 & 4.50 & 5.00 & 100 & 50 & 100 & 100 & IC \\
\hline 200 & 2000 std. & 0.50 & 4.50 & 5.00 & 200 & 100 & 200 & 100 & 200 \\
\hline 500 & 5000 std. & 0.50 & 4.50 & 5.00 & 500 & 250 & 500 & 500 & 500 \\
\hline 1000 & W.S.I & 0.50 & 4.50 & 5.00 & 1000 & 500 & 1000 & 1000 & 1000 \\
\hline 2000 & W.S.I & 1.00 & 4.00 & 5.00 & 2000 & 1000 & 2000 & 1000 & 2000 \\
\hline 5000 & W.S.I & 2.00 & 2.00 & 4.00 & 5000 & 2500 & 5000 & 5000 & 5000 \\
\hline 1000 & W.S.I & 5.00 & 0.00 & 5.00 & 10000 & 5000 & 10000 & 10000 & 10000 \\
\hline
\end{tabular}

1 The numbers in this column are target concentrations for the standard calibration curve. Target concentrations of 135TNB are one half the values shown in this column. The actual concentrations are calculated from the standard weights and are shown in the right hand columns.

${ }^{2}$ W.S. I refers to Working stock I as given previously. The dilution solution was methanol. 


\section{B. Instrument Calibration}

Standards are analyzed with a Beckman System Gold HPLC System. The system is capable of automatic tuning including lamp calibration and autnzeroing of baseline. The system automatically sets the baseline at the base of each peak and calculates the peak area and height. Detector output which produces each chromatogram is stored on computer disk. A hard copy of each chromatogram is also printed out At the conclusion of the analysis, the resulting chromatograms are studied and peak quantification parameters are optimized such that baseline placement is accurate and consistent for all samples.

\section{Preparation of Spike Solutions}

\section{A. Concentrated Spike Stock Solutions}

An individual spike stock solution is prepared for each of the analytes. An amount of analyte was weighed out so that the final concentration of the solution was $1600 \mathrm{mg} / \mathrm{L}$ for most analytes. For example, approximately $40 \mathrm{mg}$ of 246TNT was weighed out and dissolved in 25 $\mathrm{mL}$ of HPLC grade ACN in a volumetric flask. These solutions were stored in the dark (wrapped in aluminum foil) in the freezer (about $-10^{\circ}$ C). The shelf life for these solutions is expected to be approximately 6 months.

CONCENTRATED SPIKE STOCK SOLUTIONS

\begin{tabular}{lccc} 
Analyte & $\begin{array}{c}\text { Weight } \\
(\mathrm{mg})\end{array}$ & $\begin{array}{c}\text { Volume ACN } \\
(\mathrm{ml})\end{array}$ & $\begin{array}{c}\text { Actual Conc. } \\
(\mathrm{mg} / \mathrm{L})\end{array}$ \\
\hline RDX & 40.0 & 25 & 1600 \\
135TNB & 20.0 & 25 & 800 \\
246TNT & 40.0 & 25 & 1600 \\
26DNT & 40.0 & 25 & 1600 \\
24DNT & 40.0 & 25 & 1600 \\
\hline
\end{tabular}




\section{B. Working Spike Stock Solutions}

The working spike stock solutions contain all of the analytes of interest. Working spike stock $I$, used for the high level spike, was prepared by mixing equal volumes of each of the five spike stock solutions together. Thus, the final concentration of the Working Spike Stock Solution I is approximately $320 \mathrm{mg} / \mathrm{L}$ for RDX, 246TNT, 2,6DNT, 24DNT; 160 $\mathrm{mg} / \mathrm{L}$ for 135TNB. This solution was stored in the freezer and has a shelf life of 2 weeks. An appropriate volume of this working spike solution was added to the soil for spiking studies. The actual values follow.

\section{WORKING SPIKE STOCK I}

\begin{tabular}{llcc}
\hline Analyte & Stock Conc. & $\begin{array}{c}\text { Dilution of Conc. } \\
\text { Spike Soln. }\end{array}$ & $\begin{array}{c}\text { Final Conc. in } \\
\text { Working Spike Stock I } \\
(\mathrm{mg} / \mathrm{L})\end{array}$ \\
\hline (mg/L) & & 320 \\
\hdashline RDX & 1600 & $1: 5$ & 160 \\
246TNB & 800 & $1: 5$ & 320 \\
26DNT & 1600 & $1: 5$ & 320 \\
24DNT & 1600 & $1: 5$ & 320 \\
\hline
\end{tabular}

Working Spike Stock Solution II, used for the low level spike, was prepared by mixing $5 \mathrm{ml}$ of each of the five Spike Stock Solutions together and diluting this mixture to $100 \mathrm{ml}$. Thus the final concentration of Working Spike Stock Solution II is approximately $80 \mathrm{mg} / \mathrm{L}$ for RDX, 246TNT, 2,6DNT, 24DNT; $40 \mathrm{mg} / \mathrm{L}$ for $135 \mathrm{TNB}$.

\section{WORKING SPIKE STOCK II}

\begin{tabular}{llcc}
\hline Analyte & Stock Conc. & $\begin{array}{c}\text { Dilution of Conc. } \\
\text { Spike Soln. }\end{array}$ & $\begin{array}{c}\text { Forkinal Conc. in Spike Stock I } \\
\text { (mg/L) }\end{array}$ \\
& $(\mathrm{mg} / \mathrm{L})$ & & 80 \\
\hdashline RDX & 1600 & $1: 20$ & 40 \\
135TNB & 800 & $1: 20$ & 80 \\
246TNT & 1600 & $1: 20$ & 80 \\
26DNT & 1600 & $1: 20$ & 80 \\
\hline 24DNT & 1600 & $1: 20$ & \\
\hline
\end{tabular}




\section{Soil Spiking Solutions}

Soils were spiked using the working spike stock solutions shown above. The amounts of the various spike stock solutions and the dilutions used are given in the table below.

\begin{tabular}{|ccccccccc|}
\hline Level & $\begin{array}{c}\text { Spike } \\
\text { Soln. }\end{array}$ & $\begin{array}{c}\text { Volume } \\
\text { (uL) }\end{array}$ & $\begin{array}{c}\text { Soil } \\
\text { (g) }\end{array}$ & \multicolumn{4}{c|}{ Concentration Soil Basis (ug/g) } \\
& & & & RDX & $135-$ & $246-$ & 26- & 24- \\
\hline Blank & -- & 0 & 0.0 & 0.0 & 0.0 & 0.0 & 0.0 & 0.0 \\
L & WSSII & 25 & 2.0 & 1.0 & 0.5 & 1.0 & 1.0 & 1.0 \\
M & WSSII & 250 & 2.0 & 10.0 & 5.0 & 10.0 & 10.0 & 10.0 \\
H & WSSI & 500 & 2.0 & 80.0 & 40.0 & 80.0 & 80.0 & 80.0 \\
\hline
\end{tabular}

' If expressed on a solution concentration assuming a 1:10 soil to extracting solution the values would be $1 / 10$ of those shown in this table. After spiking, soil was allowed to equilibrate for 1 hour. Extraction followed using the method described in Section VII $A$.

\section{SAMPLE HANDLING STORAGE}

\section{A. Sampling Procedure}

Detailed sampling procedure is described in the Project QA Program. Field sampling involves coring with a hydraulic probe and separating the corings into the desired depth samples. All sampling equipment is cleaned with a high pressure, hot water cleaner between samples. Immediately after processing in the field samples are placed in a cooler with blue ice and transported to the laboratory.

\section{B. Containers}

Soil samples are placed in amber glass bottles that have been cleaned according to the protocol outlined in the USATHAMA QA Program. Bottle cleaning includes wash and scrub with detergent, rinse with distilled water, rinse with acetone, rinse with methylene chloride, rinse with hexane, airdrying, heat to $200^{\circ} \mathrm{C}$, cooling and caping with clean caps with Teflon liners. Bottle caps are also washed with detergent and rinsed with distilled water and dried at $40^{\circ} \mathrm{C}$. The Teflon liners are washed with detergent, rinsed with 
distilled water, rinsed with acetone, rinsed with hexane, air dried, heated for 2 hours, allowed to cool and then placed in the lid and used to cap clean bottles.

\section{Storage Conditions}

As described samples are processed in the field and placed immediately in a cooler with blue ice. Samples are then be transported to the laboratory as soon as feasible (within $\mathbf{4 8}$ hours) and stored in a freezer in the laboratory until extracted. Samples are in the field and in the laboratory to maintain sample integrity.

\section{Holding Time Limits}

All soil samples are extracted within 7 days of sampling and will be . analyzed for explosives within $\mathbf{4 0}$ days of sampling. Both samples and extracts are stored in a freezer until extracted and analyzed.

VII. Procedure

\section{A. Separations}

The general method for explosives analysis in soils we are using is a modification of the procedure proposed by Jenkins (personal communication). The modified procedure can be outlined as follows:

1. $2.00 \mathrm{~g}$ of soil and $20.0 \mathrm{~mL}$ of acetonitrile are placed in a $2.5 \mathrm{~cm} \times 20$ $\mathrm{cm}$ glass screw-cap test tube.

2. Soil is dispersed with a vortex mixer for approximately $1 \mathrm{~min}$.

3. Tubes are placed in a water bath at $22^{\circ} \mathrm{C}$ and sonicated for $18 \mathrm{hrs}$.

4. A $10.0 \mathrm{~mL}$ aliquot is combined with $10.0 \mathrm{~mL}$ of a $2.0 \mathrm{~g} / \mathrm{L}$ aqueous $\mathrm{CaCl}_{2}$ solution. The mixture is shaken and left standing for $15 \mathrm{~min}$.

5. An aliquot of the supernatant is filtered through a 0.2 um nylon filter.

6. $50 \mu \mathrm{L}$ of the filtered extract is injected into an HPLC with C-18 reverse phase guard and separating columns. The separating column is an Ultrasphere ODS $5 \mu$ column. Column lengths were 4.5 and 25.0 $\mathrm{cm}$ for the guard and separating columns, respectively. The mobile 
phase consists of $58 / 42(\mathrm{v} / \mathrm{v})$ methanol/water and has a flow rate of 1.5 $\mathrm{mL} / \mathrm{min}$. Compounds are detected at $254 \mathrm{~nm}$. Peak heights are used to quantify explosive levels in the extracts.

\section{B. Chemical reactions \\ None}

\section{References}

Jenkins, T.F., D.C. Leggett, C.L. Grant and C.F. Bauer. 1986. Reversedphase high-performance liquid chromatographic determination of nitroorganics in munitions waste water. Analytical Chemistry 58: 170-175. 

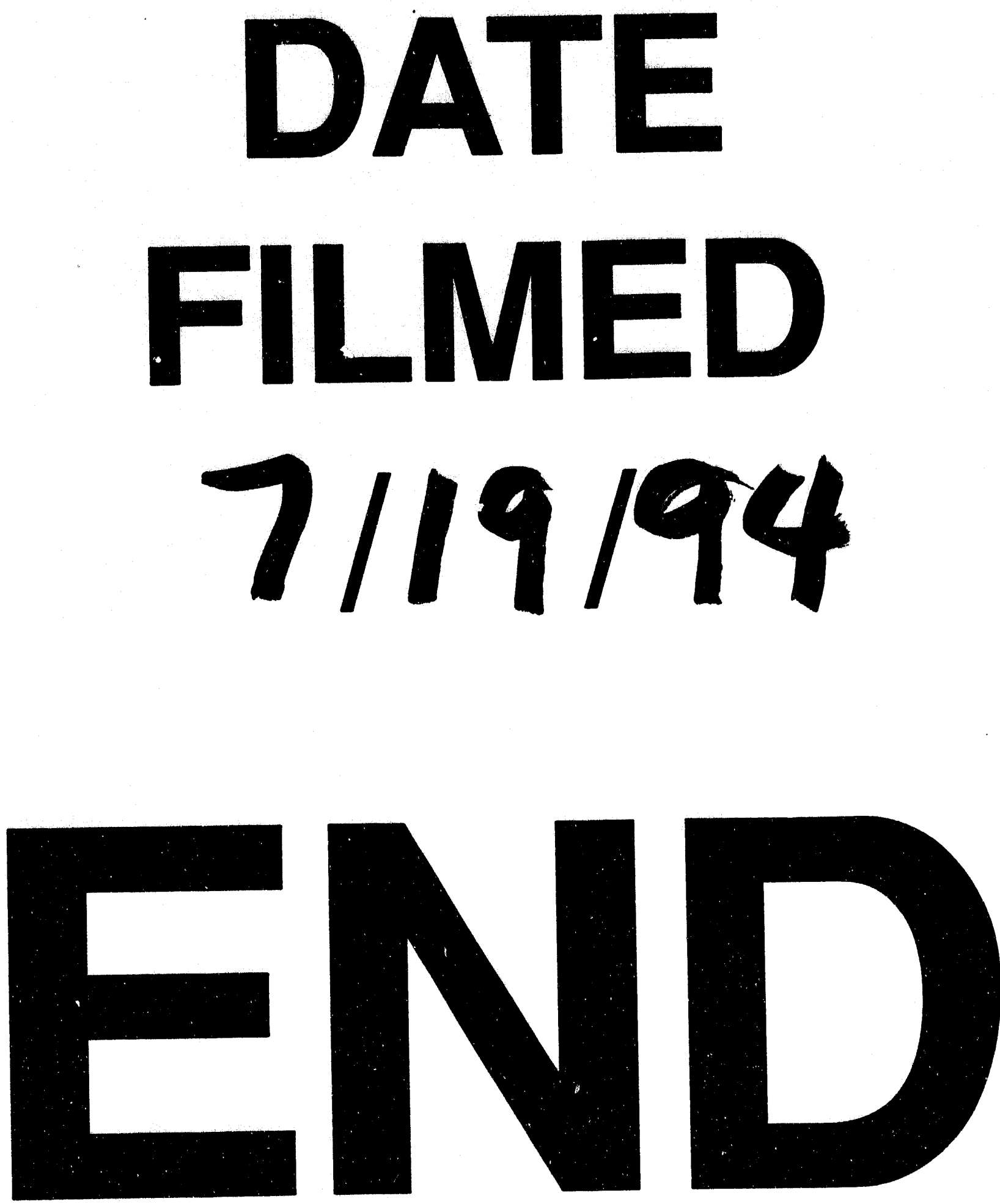


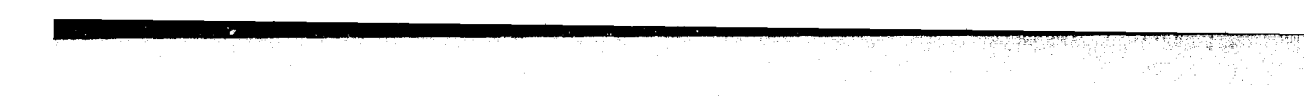

